\title{
MULTIFREQUENCY STUDIES OF THE PECULIAR QUASAR 4C +21.35 DURING THE 2010 FLARING ACTIVITY
}

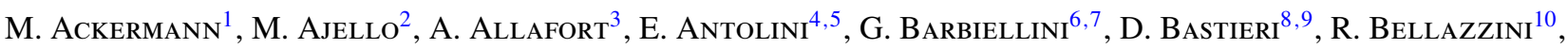

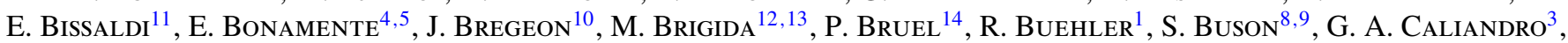

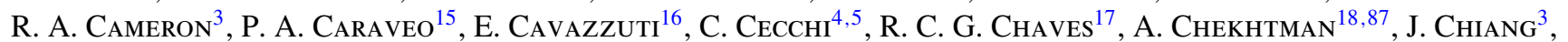
G. Chiaro ${ }^{9}$, S. Ciprini ${ }^{16,19}$, R. Clauss ${ }^{3}$, J. Cohen-Tanugi ${ }^{20}$, J. Conrad ${ }^{21,22,23,88}$, S. Cutini ${ }^{16,19}$, F. D’ Ammando ${ }^{24}$, F. De Palma ${ }^{12,13}$, C. D. Dermer ${ }^{25}$, E. do Couto e Silva ${ }^{3}$, D. Donato ${ }^{26,27}$, P. S. Drell ${ }^{3}$, C. Favuzzi ${ }^{12,13}$, J. Finke ${ }^{25}$,

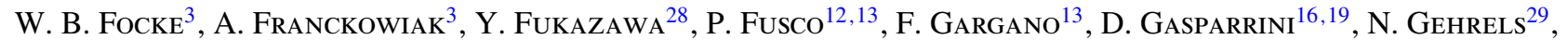
N. Giglietto ${ }^{12,13}$, F. Giordano ${ }^{12,13}$, M. Giroletti ${ }^{24}$, G. Godfrey ${ }^{3}$, I. A. Grenier ${ }^{17}$, S. Guiriec ${ }^{29,89}$, M. HaYAShida ${ }^{30}$,

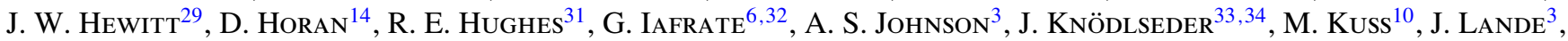

S. Larsson ${ }^{21,22,35}$, L. Latronico ${ }^{36}$, F. Longo ${ }^{6,7}$, F. Loparco ${ }^{12,13}$, M. N. Lovellette ${ }^{25}$, P. Lubrano ${ }^{4,5}$, M. MaYeR ${ }^{1}$,

M. N. Mazziotta ${ }^{13}$, J. E. McEnery ${ }^{29,27}$, P. F. Michelson ${ }^{3}$, T. Mizuno ${ }^{37}$, M. E. Monzani ${ }^{3}$, A. Morselli ${ }^{38}$,

I. V. Moskalenko ${ }^{3}$, S. Murgia ${ }^{39}$, R. Nemmen ${ }^{29}$, E. Nuss ${ }^{20}$, T. Ohsugi ${ }^{37}$, M. Orienti ${ }^{24}$, E. Orlando ${ }^{3}$, J. S. Perkins ${ }^{29}$, M. Pesce-Rollins ${ }^{10}$, F. Piron ${ }^{20}$, G. Pivato ${ }^{9}$, T. A. Porter ${ }^{3}$, S. Rainò ${ }^{12,13}$, M. Razzano ${ }^{10,90}$, A. Reimer ${ }^{3,40}$, O. Reimer $^{3,40}$,

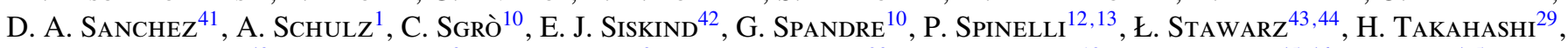

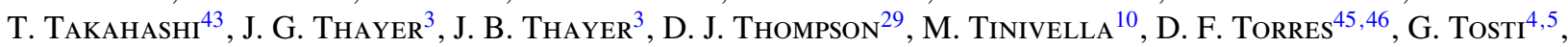

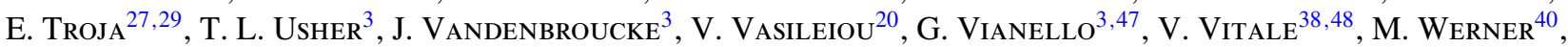
B. L. WINER ${ }^{31}$, D. L. WoOd ${ }^{49,87,89}$, K. S. WoOD ${ }^{25}$

(the Fermi Large Area Telescope Collaboration)

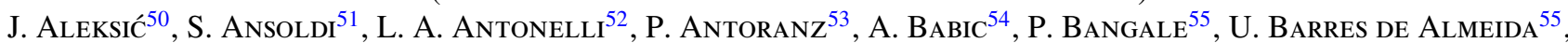
J. A. Barrio ${ }^{56}$, J. Becerra González ${ }^{57}$, W. BednareK ${ }^{58}$, K. Berger ${ }^{57}$, E. Bernardini ${ }^{1}$, A. Biland ${ }^{59}$, O. Blanch ${ }^{50}$,

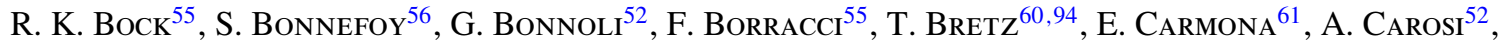

D. Carreto Fidalgo ${ }^{60}$, P. Colin ${ }^{55}$, E. Colombo ${ }^{57}$, J. L. Contreras ${ }^{56}$, J. Cortina ${ }^{50}$, S. Covino ${ }^{52}$, P. Da Vela ${ }^{53}$, F. Dazzi ${ }^{8,9}$, A. De Angelis ${ }^{51}$, G. De Caneva ${ }^{1}$, B. De Lotto ${ }^{51}$, C. Delgado Mendez ${ }^{61}$, M. Doert ${ }^{62}$, A. Domínguez ${ }^{63,91}$,

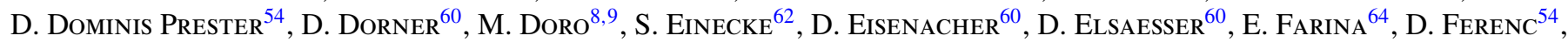
M. V. FonseCa ${ }^{56}$, L. Font ${ }^{65}$, K. Frantzen ${ }^{62}$, C. FruCK $^{55}$, R. J. García LóPez ${ }^{57}$, M. GarCZarCZyK ${ }^{57}$, D. Garrido Terrats ${ }^{65}$, M. Gaug ${ }^{65}$, G. Giavitto ${ }^{50}$, N. Godinović ${ }^{54}$, A. González Muñoz ${ }^{50}$, S. R. Gozzini ${ }^{1}$, D. Hadasch ${ }^{46}$, A. Herrero ${ }^{57}$,

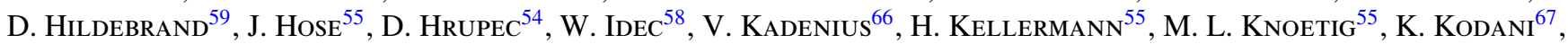

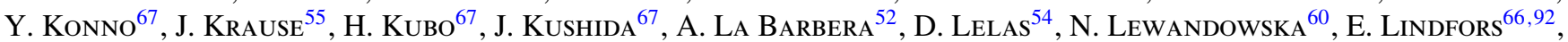

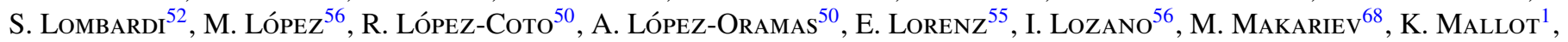

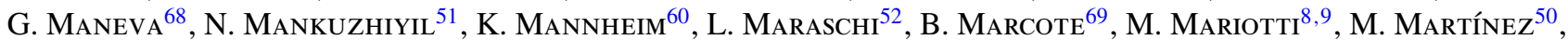
D. Mazin ${ }^{55}$, U. Menzel ${ }^{55}$, M. Meucci ${ }^{53}$, J. M. Miranda ${ }^{53}$, R. Mirzoyan ${ }^{55}$, A. Moralejo ${ }^{50}$, P. Munar-Adrover ${ }^{69}$,

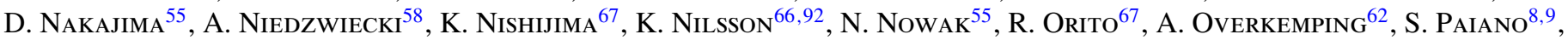
M. Palatiello ${ }^{51}$, D. Paneque ${ }^{55}$, R. Paoletti ${ }^{53}$, J. M. Paredes ${ }^{69}$, X. Paredes-Fortuny ${ }^{69}$, S. Partini ${ }^{53}$, M. Persic ${ }^{11,51}$, F. Prada ${ }^{63,95}$, P. G. Prada Moroni ${ }^{70}$, E. Prandini ${ }^{8,9}$, S. Preziuso ${ }^{53}$, I. PuljaK ${ }^{54}$, R. Reinthal ${ }^{66}$, W. Rhode ${ }^{62}$, M. Ribó ${ }^{69}$, J. Rico ${ }^{50}$, J. Rodriguez Garcia ${ }^{55}$, S. RÜGamer ${ }^{60}$, A. SAGGion ${ }^{8,9}$, T. SAito ${ }^{67}$, K. Saito ${ }^{67}$, M. SAlvati ${ }^{52}$, K. SATAleCKa ${ }^{56}$,

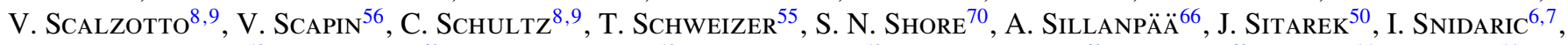
D. Sobczynska ${ }^{58}$, F. Spanier ${ }^{60}$, V. Stamatescu ${ }^{50}$, A. Stamerra ${ }^{52}$, T. Steinbring ${ }^{60}$, J. Storz ${ }^{60}$, S. Sun $^{55}$, T. Surić ${ }^{54}$,

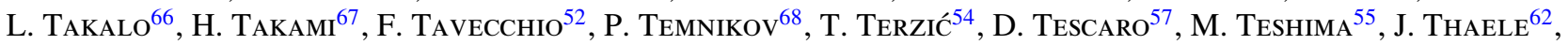

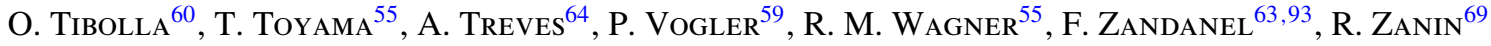

(THE MAGIC COLlaboration)

AND

M. F. Aller ${ }^{71}$, E. Angelakis ${ }^{72}$, D. A. Blinov ${ }^{73}$, S. G. DJorgovski ${ }^{74}$, A. J. DraKe ${ }^{74}$, N. V. Efimova ${ }^{73,75}$, M. A. GurWELL ${ }^{76}$,

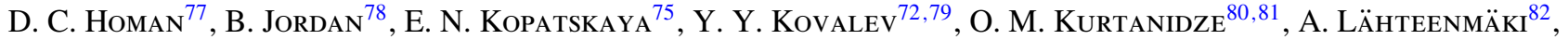

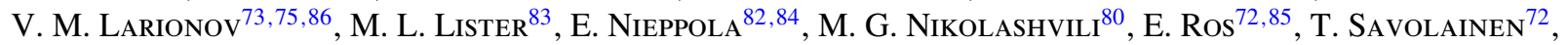

L. A. Sigua ${ }^{80}$, AND M. ToRnIKOSKI ${ }^{82}$

${ }^{1}$ Deutsches Elektronen Synchrotron DESY, D-15738 Zeuthen, Germany

${ }^{2}$ Space Sciences Laboratory, 7 Gauss Way, University of California, Berkeley, CA 94720-7450, USA

${ }^{3}$ W. W. Hansen Experimental Physics Laboratory, Kavli Institute for Particle Astrophysics and Cosmology, Department of Physics and SLAC National Accelerator Laboratory, Stanford University, Stanford, CA 94305, USA

${ }_{5}^{4}$ Dipartimento di Fisica, Università degli Studi di Perugia, I-06123 Perugia, Italy

${ }^{5}$ Istituto Nazionale di Fisica Nucleare, Sezione di Perugia, I-06123 Perugia, Italy

${ }^{6}$ Istituto Nazionale di Fisica Nucleare, Sezione di Trieste, I-34127 Trieste, Italy ${ }^{7}$ Dipartimento di Fisica, Università di Trieste, I-34127 Trieste, Italy

${ }^{8}$ Istituto Nazionale di Fisica Nucleare, Sezione di Padova, I-35131 Padova, Italy

${ }^{9}$ Dipartimento di Fisica e Astronomia “G. Galilei,” Università di Padova, I-35131 Padova, Italy

${ }^{10}$ Istituto Nazionale di Fisica Nucleare, Sezione di Pisa, I-56127 Pisa, Italy

${ }^{11}$ Istituto Nazionale di Fisica Nucleare, Sezione di Trieste, and Università di Trieste, I-34127 Trieste, Italy

${ }^{12}$ Dipartimento di Fisica "M. Merlin” dell’Università e del Politecnico di Bari, I-70126 Bari, Italy 
${ }^{13}$ Istituto Nazionale di Fisica Nucleare, Sezione di Bari, I-70126 Bari, Italy

${ }^{14}$ Laboratoire Leprince-Ringuet, École polytechnique, CNRS/IN2P3, Palaiseau, France

${ }^{15}$ INAF-Istituto di Astrofisica Spaziale e Fisica Cosmica, I-20133 Milano, Italy

${ }^{16}$ Agenzia Spaziale Italiana (ASI) Science Data Center, I-00044 Frascati (Roma), Italy

${ }^{17}$ Laboratoire AIM, CEA-IRFU/CNRS/Université Paris Diderot, Service d'Astrophysique, CEA Saclay, F-91191 Gif sur Yvette, France

${ }^{18}$ Center for Earth Observing and Space Research, College of Science, George Mason University, Fairfax, VA 22030, USA

${ }^{19}$ Istituto Nazionale di Astrofisica - Osservatorio Astronomico di Roma, I-00040 Monte Porzio Catone (Roma), Italy

${ }^{20}$ Laboratoire Univers et Particules de Montpellier, Université Montpellier 2, CNRS/IN2P3, Montpellier, France

${ }^{21}$ Department of Physics, Stockholm University, AlbaNova, SE-106 91 Stockholm, Sweden

22 The Oskar Klein Centre for Cosmoparticle Physics, AlbaNova, SE-106 91 Stockholm, Sweden

${ }^{23}$ The Royal Swedish Academy of Sciences, Box 50005, SE-104 05 Stockholm, Sweden

${ }^{24}$ INAF Istituto di Radioastronomia, I-40129 Bologna, Italy; dammando@ira.inaf.it

${ }^{25}$ Space Science Division, Naval Research Laboratory, Washington, DC 20375-5352, USA; justin.finke@ nrl.navy.mil

${ }^{26}$ Center for Research and Exploration in Space Science and Technology (CRESST) and NASA Goddard Space Flight Center, Greenbelt, MD 20771, USA; davide.donato-1@ nasa.gov

${ }^{27}$ Department of Physics and Department of Astronomy, University of Maryland, College Park, MD 20742, USA

${ }^{28}$ Department of Physical Sciences, Hiroshima University, Higashi-Hiroshima, Hiroshima 739-8526, Japan

${ }^{29}$ NASA Goddard Space Flight Center, Greenbelt, MD 20771, USA

${ }^{30}$ Institute for Cosmic-Ray Research, University of Tokyo, 5-1-5 Kashiwanoha, Kashiwa, Chiba, 277-8582, Japan

${ }^{31}$ Department of Physics, Center for Cosmology and Astro-Particle Physics, The Ohio State University, Columbus, OH 43210, USA

${ }^{32}$ Osservatorio Astronomico di Trieste, Istituto Nazionale di Astrofisica, I-34143 Trieste, Italy

${ }^{33}$ CNRS, IRAP, F-31028 Toulouse cedex 4, France

${ }^{34}$ GAHEC, Université de Toulouse, UPS-OMP, IRAP, Toulouse, France

${ }^{35}$ Department of Astronomy, Stockholm University, SE-106 91 Stockholm, Sweden

${ }^{36}$ Istituto Nazionale di Fisica Nucleare, Sezione di Torino, I-10125 Torino, Italy

${ }^{37}$ Hiroshima Astrophysical Science Center, Hiroshima University, Higashi-Hiroshima, Hiroshima 739-8526, Japan

${ }^{38}$ Istituto Nazionale di Fisica Nucleare, Sezione di Roma "Tor Vergata," I-00133 Roma, Italy

${ }^{39}$ Center for Cosmology, Physics and Astronomy Department, University of California, Irvine, CA 92697-2575, USA

${ }^{40}$ Institut für Astro- und Teilchenphysik and Institut für Theoretische Physik, Leopold-Franzens-Universität Innsbruck, A-6020 Innsbruck, Austria

${ }_{41}$ Max-Planck-Institut für Kernphysik, D-69029 Heidelberg, Germany

${ }^{42}$ NYCB Real-Time Computing Inc., Lattingtown, NY 11560-1025, USA

${ }^{43}$ Institute of Space and Astronautical Science, JAXA, 3-1-1 Yoshinodai, Chuo-ku, Sagamihara, Kanagawa 252-5210, Japan

${ }^{44}$ Astronomical Observatory, Jagiellonian University, 30-244 Kraków, Poland

45 Institut de Ciències de l'Espai (IEEE-CSIC), Campus UAB, E-08193 Barcelona, Spain

${ }^{46}$ Institució Catalana de Recerca i Estudis Avançats (ICREA), Barcelona, Spain

${ }^{47}$ Consorzio Interuniversitario per la Fisica Spaziale (CIFS), I-10133 Torino, Italy

48 Dipartimento di Fisica, Università di Roma "Tor Vergata," I-00133 Roma, Italy

${ }^{49}$ Praxis Inc., Alexandria, VA 22303

${ }^{50}$ IFAE, Edifici Cn., Campus UAB, E-08193 Bellaterra, Spain

${ }^{51}$ Dipartimento di Fisica, Università di Udine and Istituto Nazionale di Fisica Nucleare, Sezione di Trieste, Gruppo Collegato di Udine, I-33100 Udine, Italy

52 INAF National Institute for Astrophysics, I-00136 Rome, Italy

${ }^{53}$ Università di Siena, and INFN Pisa, I-53100 Siena, Italy

${ }^{54}$ Croatian MAGIC Consortium, Rudjer Boskovic Institute, University of Rijeka and University of Split, HR-10000 Zagreb, Croatia; tterzic@uniri.hr

${ }^{55}$ Max-Planck-Institut für Physik, D-80805 München, Germany

${ }^{56}$ Universidad Complutense, E-28040 Madrid, Spain

${ }^{57}$ Inst. de Astrofísica de Canarias, E-38200 La Laguna, Tenerife, Spain; jbecerragonzalez@ gmail.com

${ }^{58}$ University of Łódź, PL-90236 Lodz, Poland

${ }^{59}$ ETH Zurich, CH-8093 Zurich, Switzerland

${ }^{60}$ Universität Würzburg, D-97074 Würzburg, Germany

${ }^{61}$ Centro de Investigaciones Energéticas, Medioambientales y Tecnológicas, E-28040 Madrid, Spain

${ }^{62}$ Technische Universität Dortmund, D-44221 Dortmund, Germany

${ }^{63}$ Inst. de Astrofísica de Andalucía (CSIC), E-18080 Granada, Spain

${ }^{64}$ Università dell' Insubria, Como, I-22100 Como, Italy

${ }^{65}$ Unitat de Física de les Radiacions, Departament de Física, and CERES-IEEC, Universitat Autònoma de Barcelona, E-08193 Bellaterra, Spain

66 Tuorla Observatory, University of Turku, FI-21500 Piikkiö, Finland

${ }^{67}$ Japanese MAGIC Consortium, Division of Physics and Astronomy, Kyoto University, Japan

${ }^{68}$ Inst. for Nucl. Research and Nucl. Energy, BG-1784 Sofia, Bulgaria

${ }^{69}$ Universitat de Barcelona (ICC/IEEC), E-08028 Barcelona, Spain

${ }^{70}$ Università di Pisa, and INFN Pisa, I-56126 Pisa, Italy

${ }^{71}$ Department of Astronomy, University of Michigan, Ann Arbor, MI 48109-1042, USA

${ }^{72}$ Max-Planck-Institut für Radioastronomie, Auf dem Hügel 69, D-53121 Bonn, Germany

${ }^{73}$ Pulkovo Observatory, 196140 St. Petersburg, Russia

${ }^{74}$ Cahill Center for Astronomy and Astrophysics, California Institute of Technology, Pasadena, CA 91125, USA

${ }^{75}$ Astronomical Institute, St. Petersburg State University, St. Petersburg, Russia

${ }^{76}$ Harvard-Smithsonian Center for Astrophysics, Cambridge, MA 02138, USA

${ }^{77}$ Department of Physics, Denison University, Granville, OH 43023, USA

${ }^{78}$ School of Cosmic Physics, Dublin Institute for Advanced Studies, Dublin 2, Ireland

79 Astro Space Center of the Lebedev Physical Institute, 117997 Moscow, Russia

${ }^{80}$ Abastumani Observatory, Mt. Kanobili, 0301 Abastumani, Georgia

${ }^{81}$ Engelhardt Astronomical Observatory, Kazan Federal University, Tatarstan, Russia

82 Aalto University Metsähovi Radio Observatory, FIN-02540 Kylmala, Finland

${ }^{83}$ Department of Physics, Purdue University, West Lafayette, IN 47907, USA

${ }^{84}$ Finnish Centre for Astronomy with ESO (FINCA), University of Turku, FI-21500 Piikiiö, Finland

${ }^{85}$ Universitat de València, E-46010 València, Spain

${ }^{86}$ Isaac Newton Institute of Chile, St. Petersburg Branch, St. Petersburg, Russia

Received 2013 December 18; accepted 2014 March 26; published 2014 April 25 


\begin{abstract}
The discovery of rapidly variable Very High Energy (VHE; $E>100 \mathrm{GeV}$ ) $\gamma$-ray emission from $4 \mathrm{C}+21.35$ (PKS 1222+216) by MAGIC on 2010 June 17, triggered by the high activity detected by the Fermi Large Area Telescope (LAT) in high energy (HE; $E>100 \mathrm{MeV}) \gamma$-rays, poses intriguing questions on the location of the $\gamma$-ray emitting region in this flat spectrum radio quasar. We present multifrequency data of $4 \mathrm{C}+21.35$ collected from centimeter to VHE during 2010 to investigate the properties of this source and discuss a possible emission model. The first hint of detection at VHE was observed by MAGIC on 2010 May 3, soon after a $\gamma$-ray flare detected by Fermi-LAT that peaked on April 29. The same emission mechanism may therefore be responsible for both the HE and VHE emission during the 2010 flaring episodes. Two optical peaks were detected on 2010 April 20 and June 30, close in time but not simultaneous with the two $\gamma$-ray peaks, while no clear connection was observed between the $\mathrm{X}$-ray and $\gamma$-ray emission. An increasing flux density was observed in radio and $\mathrm{mm}$ bands from the beginning of 2009, in accordance with the increasing $\gamma$-ray activity observed by Fermi-LAT, and peaking on 2011 January 27 in the $\mathrm{mm}$ regime $(230 \mathrm{GHz}$ ). We model the spectral energy distributions (SEDs) of $4 \mathrm{C}+21.35$ for the two periods of the VHE detection and a quiescent state, using a one-zone model with the emission coming from a very compact region outside the broad line region. The three SEDs can be fit with a combination of synchrotron self-Compton and external Compton emission of seed photons from a dust torus, changing only the electron distribution parameters between the epochs. The fit of the optical/UV part of the spectrum for 2010 April 29 seems to favor an inner disk radius of $<$ six gravitational radii, as one would expect from a prograde-rotating Kerr black hole.
\end{abstract}

Key words: galaxies: active - gamma rays: general - quasars: general - quasars: individual $(4 \mathrm{C}+21.35)-$ radiation mechanisms: non-thermal

Online-only material: color figures

\section{INTRODUCTION}

In the last few years, flat spectrum radio quasars (FSRQs) have been established as a distinct Very High Energy (VHE) $\gamma$-ray blazar subclass. So far three FSRQs have been detected at $E>100 \mathrm{GeV}$ : 3C 279 (Albert et al. 2008), 4C +21.35 (Aleksić et al. 2011), and PKS 1510-089 (Cortina 2012; Abramowicz et al. 2013). These detections were surprising. The VHE emission from FSRQs is expected to be absorbed internally, if emitted within the broad line region (BLR), or externally, for sources located at redshifts where the emission is strongly attenuated by $\gamma \gamma$ pair production via interaction with the Extragalactic Background Light (EBL) photons. In addition, since FSRQs usually have their synchrotron peak at relatively low frequencies (i.e., infrared/optical bands rather than UV/X-ray), their corresponding inverse Compton peak should fall at photon energies less than $1 \mathrm{GeV}$, and thus a detection at VHE is not expected.

The FSRQ 4C +21.35 (also known as PKS 1222+21) has a redshift of $z=0.432$ (Osterbrock and Pogge 1987) with a peculiar bent large-scale radio structure (Saikia et al. 1993). Very

\footnotetext{
${ }^{87}$ Resident at Naval Research Laboratory, Washington, DC 20375, USA.

${ }^{88}$ Royal Swedish Academy of Sciences Research Fellow, funded by a grant from the K. A. Wallenberg Foundation.

${ }^{89}$ NASA Postdoctoral Program Fellow, USA.

${ }^{90}$ Funded by contract FIRB-2012-RBFR12PM1F from the Italian Ministry of Education, University and Research (MIUR).

${ }^{91}$ Now at Department of Physics, and Astronomy, UC Riverside, CA 92521, USA.

92 Now at Finnish Centre for Astronomy with ESO (FINCA), University of Turku, Finland.

${ }^{93}$ Now at GRAPPA Institute, University of Amsterdam, 1098XH Amsterdam, Netherlands.

${ }_{94}$ Now at Ecole polytechnique fédérale de Lausanne (EPFL), Lausanne, Switzerland.

${ }^{95}$ Also at Instituto de Fisica Teorica, UAM/CSIC, E-28049 Madrid, Spain.
}

large apparent superluminal motion $\left(\beta_{\text {app }} \sim 10-15\right)$ has been detected on milliarcsecond scales for sub-components of the jet (Jorstad et al. 2001; Homan et al. 2001). On the other hand, the ratio of the core-to-extended radio fluxes at $\mathrm{GHz}$ frequencies is of the order of unity; thus it is formally a "lobe-dominated quasar" (Kharb \& Shastri 2004; Wang et al. 2004).

In $\mathrm{GeV} \gamma$-rays, the source was in a quiescent state from the start of the Fermi Gamma-ray Space Telescope mission in 2008 August until 2009 September. After this period, a gradually increasing flux was observed, up to an interval of flaring activity in the first half of 2010. In particular, $4 C+21.35$ underwent two very strong outbursts in 2010 April and June, observed by the Large Area Telescope (LAT) on board Fermi and composed of several major flares characterized by rise and decay timescales of the order of one day (Tanaka et al. 2011). During the second flaring activity, VHE emission from 4C +21.35 was detected with the MAGIC Cherenkov telescopes on 2010 June 17, with a flux doubling time of about 10 minutes (Aleksić et al. 2011). The simultaneous Fermi-LAT and Major Atmospheric Gamma Imaging Cherenkov (MAGIC) spectrum, corrected for EBL absorption, can be described by a single power law with photon index $\Gamma_{\gamma}=2.72 \pm 0.34$ between $3 \mathrm{GeV}$ and $400 \mathrm{GeV}$, consistent with emission from a single component in the jet. The absence of a spectral cut-off for $E<130 \mathrm{GeV}$ constrains the $\gamma$-ray emission region to lie outside the BLR, which would otherwise absorb the $\approx 10-20 \mathrm{GeV}$ photons by $\gamma \gamma \rightarrow e^{ \pm}$ production when these $\gamma$-rays pass through the intense circumnuclear photon fields provided by the BLR itself. At the same time, the rapid VHE variability observed suggests an extremely compact emission region, with size $R \leqslant c t_{\mathrm{var}} \delta_{D} /(1+z) \sim$ $10^{15}\left(\delta_{D} / 80\right)\left(t_{\mathrm{var}} / 10\right.$ minutes $) \mathrm{cm}$, where $t_{\mathrm{var}}$ is the variability timescale and $\delta_{D}$ is the Doppler factor. If the blob takes up the entire cross section of the jet, it implies that the emitting region is at a distance $r \sim R / \theta_{\text {open }} \sim 5.7 \times 10^{16}\left(\delta_{D} / 80\right)\left(t_{\mathrm{var}} / 10\right.$ minutes $)$ $\left(\theta_{\text {open }} / 1 \mathrm{deg}\right)^{-1} \mathrm{~cm}$, where $\theta_{\text {open }}$ is the half-opening angle of the jet. Even for a highly relativistic jet with $\delta_{D} \sim 100$, the location of the emission region should be well within the BLR 
Table 1

Observatories Contributing to the Presented Data Set of $4 \mathrm{C}+21.35$ at Different Frequencies

\begin{tabular}{|c|c|c|}
\hline Waveband & Observatory & Frequency/Band \\
\hline Radio & $\begin{array}{c}\text { SMA } \\
\text { Metsähovi } \\
\text { VLBA (MOJAVE) } \\
\text { OVRO } \\
\text { UMRAO } \\
\text { Medicina } \\
\text { F-GAMMA }\end{array}$ & $\begin{array}{c}230 \mathrm{GHz} \\
37 \mathrm{GHz} \\
15 \mathrm{GHz} \\
15 \mathrm{GHz} \\
8.0,14.5 \mathrm{GHz} \\
5,8 \mathrm{GHz} \\
2.6,4.8,8.4,10.5,14.6,23.1,32, \\
86.2,142.3 \mathrm{GHz}\end{array}$ \\
\hline Optical & $\begin{array}{l}\text { Abastumani } \\
\text { ATOM } \\
\text { Catalina } \\
\text { Crimean } \\
\text { KVA } \\
\text { St. Petersburg } \\
\text { Steward } \\
\text { Swift-UVOT }\end{array}$ & $\begin{array}{c}R \\
R \\
V \\
R \\
R \\
R \\
V \\
v, b, u\end{array}$ \\
\hline UV & Swift-UVOT & $w 1, m 2, w 2$ \\
\hline X-rays & $\begin{array}{l}\text { Swift-XRT } \\
\text { Swift-BAT }\end{array}$ & $\begin{array}{l}0.3-10 \mathrm{keV} \\
15-50 \mathrm{keV}\end{array}$ \\
\hline $\begin{array}{l}\mathrm{HE} \gamma \text {-rays } \\
\text { VHE } \gamma \text {-rays }\end{array}$ & $\begin{array}{c}\text { Fermi-LAT } \\
\text { MAGIC }\end{array}$ & $\begin{array}{c}0.1-300 \mathrm{GeV} \\
70 \mathrm{GeV}-5 \mathrm{TeV}\end{array}$ \\
\hline
\end{tabular}

radius for $4 \mathrm{C}+21.35$, likely $R_{\mathrm{BLR}} \approx 2 \times 10^{17} \mathrm{~cm}$ (Tanaka et al. 2011).

Different models have been proposed to explain the unusual behavior of $4 \mathrm{C}+21.35$. A very narrow jet can preserve variability at the pc scale, but the likelihood of being in the beam of such a thin jet is small, unless there were many narrow jets, as in a jets-within-jet/mini-jets scenario (Ghisellini \& Tavecchio 2008; Giannios et al. 2009; Tavecchio et al. 2011). An alternative model is a compact emission region at the parsec scale responsible for the emission at higher energies, with a second zone either inside or outside the BLR to complete the modeling of the emission at lower energies (Tavecchio et al. 2011). The compact emission sites at the pc scale could be due to self-collimating jet structures (Nalewajko et al. 2012), where the magnetic field dominates the energy density, or to turbulent cells (e.g., Nalewajko et al. 2011; Marscher \& Jorstad 2010). Another possibility is that the acceleration of ultra-high energy cosmic rays protons in the inner jet leads to an outflowing beam of neutrons that deposit their energy into ultra-relativistic pairs that radiate VHE synchrotron emission at the paresec scale (Dermer et al. 2012), with associated neutrino production. Even more exotic scenarios have been proposed, such as photons produced inside the BLR, tunneling through it via photon to axion-like particle oscillations (Tavecchio et al. 2012).

In this paper, we present the multifrequency data of $4 \mathrm{C}+21.35$ collected from radio to VHE during 2010, and discuss a possible emission model for this source. A summary of the complete multiwavelength data of $4 \mathrm{C}+21.35$ presented in this paper and the relative facilities can be found in Table 1. The paper is organized as follows. In Sections 2 and 3, we briefly report the LAT and MAGIC data analysis and results, respectively. In Section 4, we report the result of Swift optical/UV/X-ray observations. Optical data collected by the Abastumani, ATOM, Catalina, Crimean, KVA, Steward, and St. Petersburg observatories are presented in Section 5. In Section 6, we present the radio and $\mathrm{mm}$ data collected by the Medicina, UMRAO,
MOJAVE, OVRO, F-GAMMA, Metsähovi, and SMA facilities. In Section 7, we discuss the light curves behavior and the spectral energy distribution (SED) modeling of three different epochs, and finally we draw our conclusions in Section 8.

Throughout the paper, a $\Lambda$ CDM cosmology with $H_{0}=$ $71 \mathrm{~km} \mathrm{~s}^{-1} \mathrm{Mpc}^{-1}, \Omega_{\Lambda}=0.73$, and $\Omega_{m}=0.27$ is adopted. The corresponding luminosity distance at $z=0.432$ is $d_{L}=$ $2370 \mathrm{Mpc}$, and 1 arcsec corresponds to a projected size of $5.6 \mathrm{kpc}$.

\section{MAGIC DATA AND ANALYSIS}

The MAGIC experiment is situated in the Observatorio del Roque de los Muchachos in the Canary Island of La Palma ( $28^{\circ} 45^{\prime}$ north, $18^{\circ} 54^{\prime}$ west), $2200 \mathrm{~m}$ above sea level. It consists of two $17 \mathrm{~m}$ Imaging Atmospheric Cherenkov Telescopes and can reach an energy threshold as low as $50 \mathrm{GeV}$ in standard trigger mode. Details on the performance of the MAGIC telescope stereo system can be found in Aleksić et al. (2012).

MAGIC observed 4C +21.35 between 2010 May 3 and June 19. In total, $16 \mathrm{hr}$ of good-quality data were collected. The data analysis was performed in the MAGIC Analysis and Reconstruction Software analysis framework (Moralejo et al. 2009; Lombardi et al. 2011). On May 3 (MJD 55319), MAGIC obtained an excess with respect to the background of $\approx 78$ events in $2.2 \mathrm{hr}$ of observation, which yielded a marginal detection with a signal significance of $4.4 \sigma$ using the Equation (17) of Li and Ma (1983). On June 17 (MJD 55364), MAGIC obtained a $\gamma$-ray excess of 190 events in a 30 minute long observation, yielding a signal significance of $10.2 \sigma$, implying the first significant detection of this source in VHE $\gamma$-rays (Aleksić et al. 2011). The VHE detection on June 17 shows fast variability with a flux doubling time of $8.6_{-0.9}^{+1.1}$ minutes, which is the fastest time variation ever observed in a FSRQ, and among the shortest time scales measured for $\mathrm{TeV}$ emitters (see, e.g., Albert et al. 2007; Aharonian et al. 2007). The observed spectrum covered the energy range from $70 \mathrm{GeV}$ up to at least $400 \mathrm{GeV}$ and can be fit with a single power law with photon index $\Gamma_{\gamma}=3.75 \pm 0.27$. The spectrum corrected for the effect of EBL absorption making use of the EBL model from Dominguez et al. (2011) can be also described by a single power law with photon index $\Gamma_{\gamma}=2.72 \pm 0.34$ between $3 \mathrm{GeV}$ and $400 \mathrm{GeV}$ (see Aleksić et al. 2011).

None of the other nights showed a statistically significant excess of signal over the background. Upper limits at 95\% C.L. were calculated above $150 \mathrm{GeV}$ assuming a power law with the same photon index measured on June 17 (i.e., $\Gamma_{\gamma}=3.75$ ) for the nights between May 5 and June 13. The rest of the nights were not included in the upper limit calculation due to strong moonlight constraints. The upper limits range between $1.4 \%$ Crab units (C.U.) (on May 30; MJD 55346) and 12.7\% C.U. (on June 12; MJD 55359), as reported in Table 2. Considering the period from May 5 to June 13 (total time: $12.5 \mathrm{hr}$ ) an upper limit of $1.6 \%$ C.U. was estimated.

\section{FERMI-LAT}

The Fermi-LAT is a $\gamma$-ray telescope operating from $20 \mathrm{MeV}$ to above $300 \mathrm{GeV}$. The LAT has a large peak effective area ( $\sim 8000 \mathrm{~cm}^{2}$ for $1 \mathrm{GeV}$ photons), a relative energy resolution typically $\sim 10 \%$, and a field of view of about 2.4 sr with an angular resolution ( $68 \%$ containment angle) better than $1^{\circ}$ for energies above $1 \mathrm{GeV}$. Further details about the LAT are given by Atwood et al. (2009). 
Table 2

Daily Upper Limits Estimated by MAGIC for $E>150 \mathrm{GeV}$ Assuming a Spectrum Slope 3.7

\begin{tabular}{lccc}
\hline \hline $\begin{array}{l}\text { Date } \\
(\mathrm{UT})\end{array}$ & $\begin{array}{c}\text { Effective Time } \\
(\mathrm{hr})\end{array}$ & $\begin{array}{c}\text { Integral Limit } \\
\left(\mathrm{cm}^{-2} \mathrm{~s}^{-1}\right) \text { above 150 GeV }\end{array}$ & $\begin{array}{c}\text { Integral Limit } \\
(\text { in MAGIC C.U.) }\end{array}$ \\
\hline 2010 May 5 & 0.5 & $1.2 \mathrm{e}-11$ & $3.7 \%$ \\
2010 May 6 & 0.7 & $1.1 \mathrm{e}-11$ & $3.3 \%$ \\
2010 May 7 & 0.8 & $1.8 \mathrm{e}-11$ & $5.5 \%$ \\
2010 May 8 & 1.4 & $1.7 \mathrm{e}-11$ & $5.4 \%$ \\
2010 May 30 & 0.9 & $4.6 \mathrm{e}-12$ & $1.4 \%$ \\
2010 May 31 & 1.0 & $2.6 \mathrm{e}-11$ & $8.1 \%$ \\
2010 Jun 1 & 1.2 & $5.1 \mathrm{e}-12$ & $1.6 \%$ \\
2010 Jun 2 & 0.9 & $1.0 \mathrm{e}-11$ & $3.7 \%$ \\
2010 Jun 3 & 1.1 & $8.4 \mathrm{e}-12$ & $2.6 \%$ \\
2010 Jun 4 & 1.2 & $8.0 \mathrm{e}-12$ & $2.5 \%$ \\
2010 Jun 6 & 1.0 & $1.4 \mathrm{e}-11$ & $4.3 \%$ \\
2010 Jun 7 & 0.6 & $2.1 \mathrm{e}-11$ & $6.4 \%$ \\
2010 Jun 8 & 0.7 & $1.3 \mathrm{e}-11$ & $4.0 \%$ \\
2010 Jun 9 & 0.9 & $2.4 \mathrm{e}-11$ & $7.3 \%$ \\
2010 Jun 12 & 0.6 & $4.1 \mathrm{e}-11$ & $12.7 \%$ \\
2010 Jun 13 & 0.6 & $2.5 \mathrm{e}-11$ & $7.8 \%$ \\
\hline
\end{tabular}

Very strong $\mathrm{GeV}$ flares from $4 \mathrm{C}+21.35$ were detected by Fermi-LAT in 2010 April and June, with spectra characterized by a broken power law with spectral breaks near $1-3 \mathrm{GeV}$ photon energies and a photon index after the break $\sim 2.4$. In contrast, the quiescent state observed by the LAT during 2008 August-2009 September has been fit by a single power law with photon index $\Gamma_{\gamma}=2.57 \pm 0.07$. All details of the LAT analysis for that period were presented in Tanaka et al. (2011). After the 2010 flaring period, a decreasing $\gamma$-ray activity of $4 \mathrm{C}+21.35$ was observed by Fermi-LAT, and then in mid-2011 the source faded back into a quiescent state. ${ }^{96} 4 \mathrm{C}+21.35$ is found in the first Fermi hard source list (1FHL) as 1FHL J1224.8+2122 (Ackermann et al. 2013). This object is the most variable source in the $1 \mathrm{FHL}$ catalog.

\section{SWIFT OBSERVATIONS}

The Swift satellite (Gehrels et al. 2004) performed 28 observations of $4 \mathrm{C}+21.35$ between 2010 February 12 and June 23 . The observations were performed with all three onboard instruments: the Burst Alert Telescope (BAT; Barthelmy et al. 2005, 15-150 keV), the X-ray Telescope (XRT; Burrows et al. 2005, 0.2-10.0 keV), and the UltraViolet Optical Telescope (UVOT; Roming et al. 2005, 170-600 nm).

\subsection{Swift/BAT}

$4 \mathrm{C}+21.35$ is detected in the BAT 70 month catalog, generated from the all-sky survey in the time period 2004 November-2010 August. The data reduction and extraction procedure of the 8 channel spectrum is described in Baumgartner et al. (2013). The 14-195 keV spectrum is well described by a power-law with photon index of $1.76_{-0.23}^{+0.25}\left(\chi_{\text {red }}^{2}=0.60,6\right.$ d.o.f. $)$. The resulting unabsorbed 14-195 keV flux is $(2.2 \pm 0.4) \times 10^{-11} \mathrm{erg} \mathrm{cm}^{-2} \mathrm{~s}^{-1}$. No significant variability was observed in the BAT light curve on monthly time scales. Nevertheless, the hard X-ray flux of this source is below the sensitivity of the BAT instrument for the short exposure times of single Swift observations.

\footnotetext{
96 http://fermi.gsfc.nasa.gov/FTP/glast/data/lat/catalogs/asp/current/ lightcurves/PKSB1222+216_86400.png
}

\subsection{Swift/XRT}

The XRT data were processed with standard procedures (xrtpipeline v0.12.6), filtering, and screening criteria by using the HEASoft package (v6.11). The data were collected in photon counting mode in all observations, and only XRT event grades $0-12$ were selected. The source count rate was low $\left(<0.5\right.$ counts $\left.\mathrm{s}^{-1}\right)$, thus pile-up correction was not required. Data collected in the same day were summed in order to have better statistics. Since the observation performed on 2010 May 26 was short $(\sim 500 \mathrm{~s})$, it was not considered. Source events were extracted from a circular region with a radius of 20 pixels (1 pixel $=2$ ".36), while background events were extracted from a circular region with radius of 50 pixels away from the source region. Ancillary response files were generated with xrtmkarf, and account for different extraction regions, vignetting and point-spread function corrections. When the number of photons collected was smaller than 200, the Cash statistic was used (Cash 1979).

We fit the spectra for all the individual Swift observations with an absorbed power law with a neutral hydrogen column density fixed to its Galactic value $\left(N_{\mathrm{H}}=2.09 \times 10^{20} \mathrm{~cm}^{-2}\right.$; Kalberla et al. 2005). The X-ray light curve and spectral shape derived from these fits is discussed in Section 7.1 together with the other multiwavelength data.

\subsection{Swift/UVOT}

The script that handles the UVOT analysis is uvotgrblc (available within HEASoft). It determines the aperture corrected magnitude by (1) selecting the aperture size based on the observed source count rate and the presence of close field sources; (2) choosing the background region based on the surface brightness among three annular regions centered on the main source in the summed images (circular regions around field sources are automatically excluded); (3) finding field stars to estimate the aperture correction, specific for each observation; and (4) calling the task uvot source to estimate the photometry.

Since $4 \mathrm{C}+21.35$ is a very bright object in the optical and UV range and lies in a sparsely populated area of the sky, uvotgrblc selected a circle of $5^{\prime \prime}$ as the source extraction region and a full annulus for the background region for all the observations. The typical inner/outer radii for the background regions were $27^{\prime \prime} / 35^{\prime \prime}$ and $35^{\prime \prime} / 42^{\prime \prime}$. The UVOT magnitudes during these observations showed ranges as follows: $v=15.67-15.21, b=15.65-15.43, u=14.67-14.34, w 1=$ $14.37-14.08, m 2=14.25-13.90, w 2=14.16-13.90$, with a typical error of $0.06 \mathrm{mag}$. As discussed in detail in Section 7.1, no significant increase in flux was observed by UVOT during 2010 February-June, but the sparse coverage does not allow us to draw firm conclusions.

\section{OPTICAL OBSERVATIONS}

In this section, we briefly describe the programs performing optical observations of $4 \mathrm{C}+21.35$ and the corresponding data analysis. These optical data are discussed together with the multiwavelength data in Section 7.1.

\subsection{Abastumani, Crimean and St. Petersburg Data}

Observational data at optical wavelengths ( $R$ band) were obtained at the $0.7 \mathrm{~m}$ reflector of the Crimean Astrophysical Observatory and $0.4 \mathrm{~m} \mathrm{LX}-200$ telescope of the Astronomical Institute of St. Petersburg State University, both equipped with 
photo polarimeters based on ST-7XME CCDs. A standard technique of bias and dark subtraction and flat-field correction was used. Photometric calibration was made relative to two nearby standard stars, located in the same field.

Optical observations in $R$-band were performed also by the $0.7 \mathrm{~m}$ meniscus $\mathrm{f} / 3$ telescope of Abastumani Astrophysical Observatory in Abastumani, Georgia.

\subsection{ATOM Data}

Optical observations in $R$ filter for this campaign were obtained between 2010 February and May with the $0.8 \mathrm{~m}$ optical telescope ATOM in Namibia (Hauser et al. 2004). ATOM is operated robotically by the H.E.S.S. collaboration and obtains automatic observations of confirmed or potential $\gamma$-bright blazars. Data analysis (debiassing, flat fielding, and photometry with Source-Extractor; Bertin and Arnouts 1996) is conducted automatically using the pipeline developed by the ATOM Team.

\subsection{Catalina Real-Time Transient Survey}

The source is monitored by the Catalina Real-Time Transient Survey (CRTS; http://crts.caltech.edu; Drake et al. 2009; Djorgovski et al. 2011), using the $0.68 \mathrm{~m}$ Schmidt telescope at Catalina Station, AZ, and an unfiltered CCD. The typical cadence is to obtain four exposures separated by 10 minutes in a given night; this may be repeated up to four times per lunation, over a period of $\sim 6-7$ months each year, while the field is observable. Photometry is obtained using the standard SourceExtractor package (Bertin and Arnouts 1996), and roughly calibrated to the $V$ band in terms of the magnitude zero point. The light curve, accessible through the CRTS Web site and spanning $\sim$ six yr, shows a dramatic increase in optical variability of this source starting in late 2009.

\subsection{KVA Data}

The KVA (Kungliga Vetenskaps Akademientelescope) is located on Roque de los Muchachos, La Palma (Canary Islands), and is operated by the Tuorla Observatory, Finland (http://users.utu.fi/kani/1m). The telescope consists of a $0.6 \mathrm{~m}$ $\mathrm{f} / 15$ Cassegrain devoted to polarimetry, and a $0.35 \mathrm{~m} \mathrm{f} / 11 \mathrm{SCT}$ auxiliary telescope for multicolor photometry. The telescope has been successfully operated remotely since autumn 2003. The KVA is used for optical support observations for MAGIC by making $R$-band photometric observations, typically one measurement per night per source. The data were reduced by the Tuorla Observatory Team as described in K. Nilsson et al. (in preparation).

\subsection{Steward Observatory}

$4 \mathrm{C}+21.35$ was systematically monitored by Steward Observatory during the Fermi observations, providing spectropolarimetry and spectrophotometry measurements of this object in $V$ band, ${ }^{97}$ as reported also in Smith et al. (2011). Figure 1 shows the behavior of the observed degree of optical polarization $\mathrm{P}$, and the position angle of the polarization vector $\Theta$ as a function of time. Visual inspection of the plot seems to show that in general periods of high flux correspond to periods of relatively high polarization degree and only small rotation of the polarization angle vector. In particular, a marginal increase of polarization degree but no significant rotation of the polarization angle was

\footnotetext{
97 http://james.as.arizona.edu/ psmith/Fermi/
}

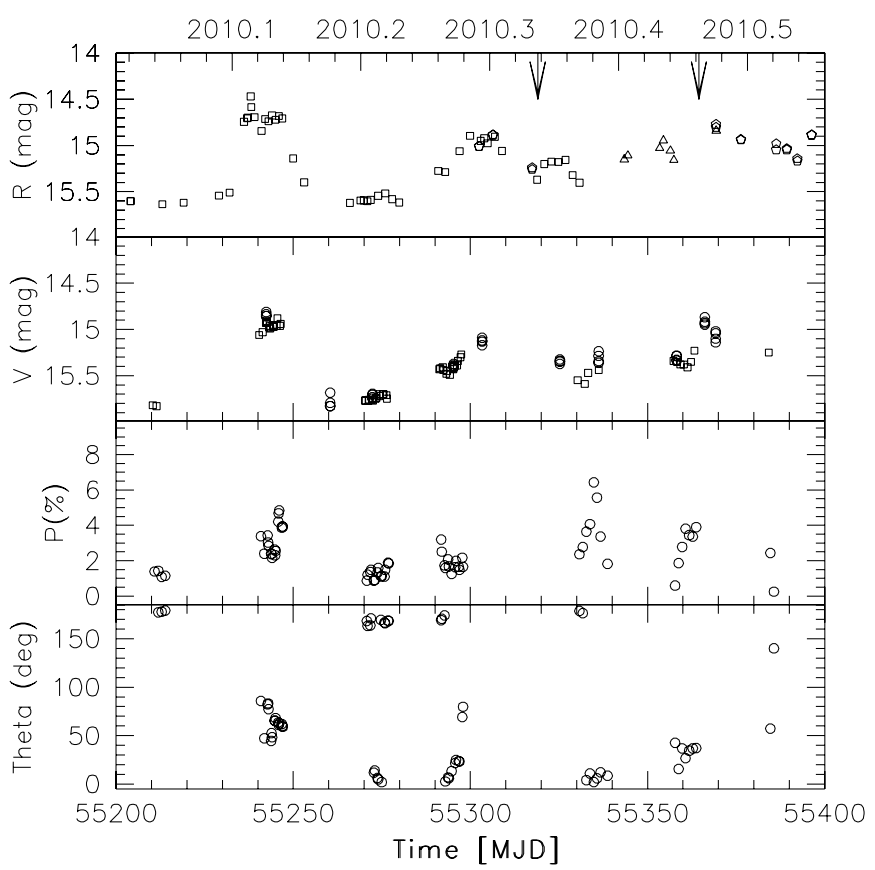

Figure 1. Optical magnitudes in the $R$ band (top panel; circles: Abastumani, squares: ATOM, triangles: KVA, pentagons: Crimean and St. Petersburg), $V$ band (second panel; circles: CRTS, squares: Steward), percentage of polarized flux (third panel) and polarization position angle in $V$ band (bottom panel) are shown. For clarity the errors (typically $<5 \%$ ) are not shown. The downward arrows indicate the times of the VHE detections by MAGIC.

observed in 2010 June, during the period of HE and VHE flaring activity.

Spectrophotometry during 2010-2011 did not show significant changes in the flux of the broad $\mathrm{Mg}$ II $\lambda 2800$ and Balmer emission lines despite large optical continuum variation. This indicates that non-thermal emission from the jet has a negligible influence on the BLR lines fluxes. Smith et al. (2011) suggested that the beaming jet emission intersects only a small fraction of the volume containing the emission-line gas. A different behavior was observed in $3 \mathrm{C} 454.3$, with a significant increase of the $\mathrm{Mg}$ II emission line flux during the optical and $\gamma$-ray flaring activity in 2010 November. This event occurred after a mm flare onset, during an increase in the optical polarization percentage, and before the emergence of a superluminal knot from the radio core. This suggests the presence of BLR clouds surrounding the radio core in 3 C 454.3 (Leon-Tavares et al. 2013).

\section{RADIO AND MM OBSERVATIONS}

In this section, we present the radio and mm light curves and spectra of 4C +21.35 collected between 2009 January 1 and 2011 February 28 to investigate their connection with the $\gamma$-ray activity. The data collected between $230 \mathrm{GHz}$ and $5 \mathrm{GHz}$ are reported in Figure 2 and discussed in detail in Section 7.1. In addition we studied the radio structure and jet kinematics of this source during the MOJAVE monitoring observations.

\subsection{SMA Data}

The $230 \mathrm{GHz}(1.3 \mathrm{~mm})$ light curve was obtained at the Submillimeter Array (SMA) on Mauna Kea (Hawaii). 4C +21.35 is included in an ongoing monitoring program at the SMA to determine the fluxes of compact extragalactic radio sources that can be used as calibrators at mm wavelengths. Details of the observations and data reduction can be found in Gurwell et al. (2007). 


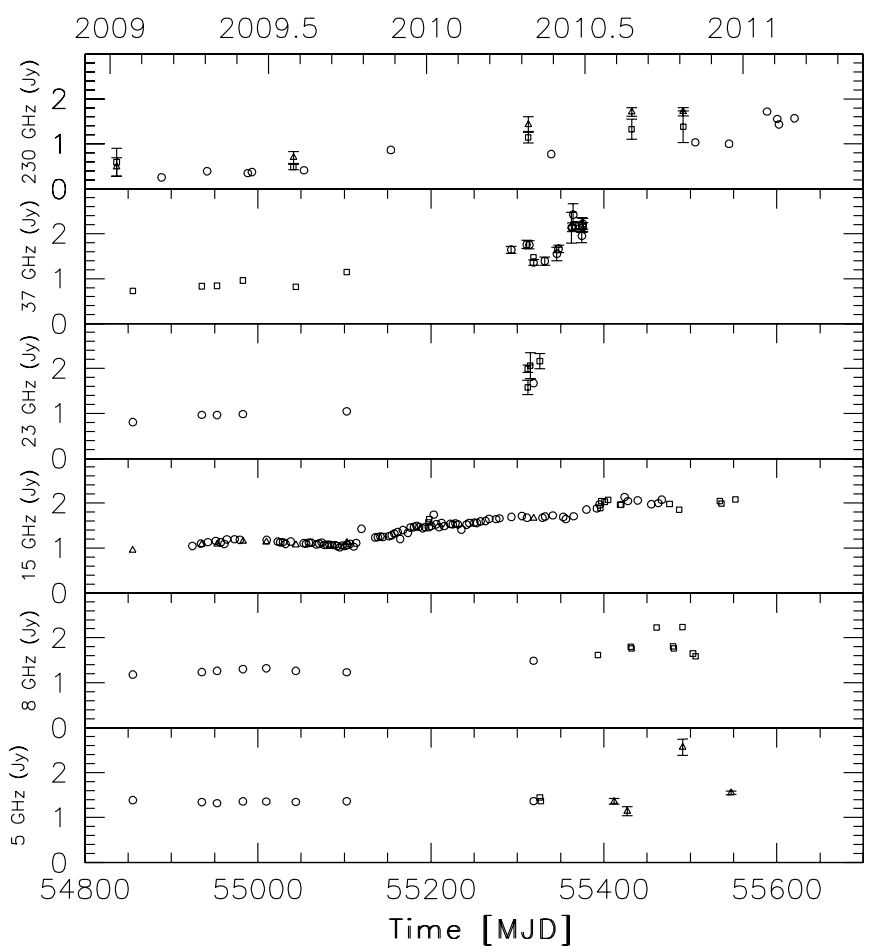

Figure 2. Radio and $\mathrm{mm}$ light curves of $4 \mathrm{C}+21.35$ in units of $\mathrm{Jy}$. The period covered is between 2009 January 1 (MJD 54837) and 2011 February 28 (MJD 55620). The data are collected (from top to bottom panel) by SMA at $230 \mathrm{GHz}$ (circles), IRAM at $142 \mathrm{GHz}$ (squares) and $86 \mathrm{GHz}$ (triangles); Metsähovi at $37 \mathrm{GHz}$ (circles) and Effelsberg at $32 \mathrm{GHz}$ (squares); Effelsberg at $23 \mathrm{GHz}$ (circles) and Medicina a $22 \mathrm{GHz}$ (squares); OVRO (circles), UMRAO (squares), Effelsberg (triangles) at $15 \mathrm{GHz}$; Effelsberg (circles) and UMRAO (squares) at $8 \mathrm{GHz}$; Effelsberg (circles), Medicina (squares), and UMRAO (triangles) at $5 \mathrm{GHz}$. For clarity, errors are not shown when $<5 \%$.

Data from this program are updated regularly and are available at the SMA Web site. ${ }^{98}$

\subsection{F-GAMMA Project}

Radio spectra and light curves of $4 \mathrm{C}+21.35$ were obtained within the framework of a Fermi-related monitoring program of $\gamma$-ray blazars (F-GAMMA project; Fuhrmann et al. 2007). The frequency range spans $2.64 \mathrm{GHz}$ to $142 \mathrm{GHz}$ using the Effelsberg $100 \mathrm{~m}$ and IRAM $30 \mathrm{~m}$ telescopes. The Effelsberg measurements were conducted with the secondary focus heterodyne receivers at $2.64,4.85,8.35,10.45,14.60,23.05$, 32.00 , and $43 \mathrm{GHz}$. The observations were performed quasisimultaneously with cross-scans, that is, slewing over the source position, in azimuth and elevation directions, with adaptive numbers of sub-scans for reaching the desired sensitivity (for details, see Fuhrmann et al. 2008; Angelakis et al. 2008). Pointing offset correction, gain correction, atmospheric opacity correction, and sensitivity correction have been applied to the data. The IRAM $30 \mathrm{~m}$ observations were carried out with calibrated cross-scans using the EMIR horizontal and vertical polarization receivers operating at 86.2 and $142.3 \mathrm{GHz}$. The opacity-corrected intensities were converted into the standard temperature scale and finally corrected for small remaining pointing offsets and systematic gain-elevation effects. The conversion to the standard flux density scale was done using the instantaneous conversion

\footnotetext{
$98 \mathrm{http} / / / \mathrm{sma1.sma.hawaii.edu/callist/callist.html.} \mathrm{Use} \mathrm{of} \mathrm{SMA} \mathrm{data} \mathrm{in}$ publication requires obtaining permission in advance.
}

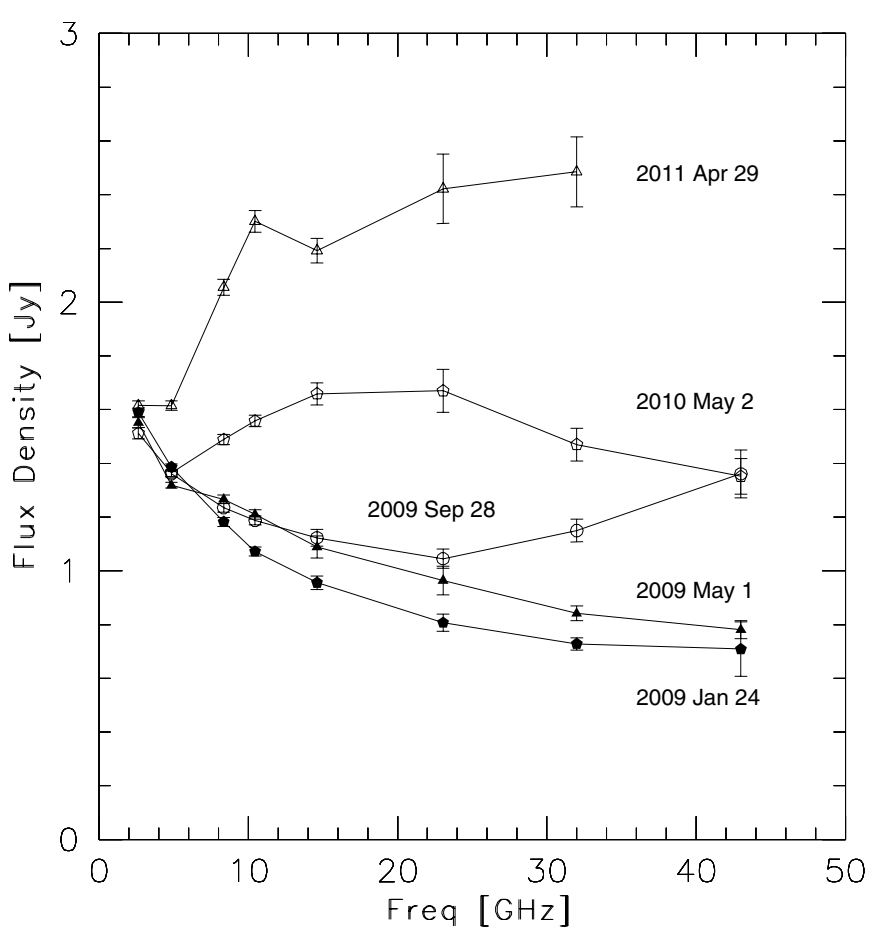

Figure 3. Radio spectra of 4C +21.35 obtained by Effelsberg on 2009 January 24 (filled pentagons), 2009 May 1 (filled triangles), 2009 September 28 (open circles), 2010 May 2 (open pentagons), and 2011 April 29 (open triangles) from 2.64 to $43 \mathrm{GHz}$.

factors derived from frequently observed primary (Mars, Uranus) and secondary (W3(OH), K350A, NGC 7027) calibrators. The radio spectra from $2.64 \mathrm{GHz}$ to $43 \mathrm{GHz}$ obtained during five epochs of Effelsberg observations between 2009 January 24 and 2011 April 29 are shown in Figure 3. A significant increase of the flux density has been observed from 2009 May to September at $43 \mathrm{GHz}$, while at longer wavelengths the increase occurs later, likely due to synchrotron self-absorption opacity effects. This time difference led to a significant radio spectral evolution, possibly related to the activity observed in $\gamma$-rays.

\subsection{Metsähovi Data}

The $37 \mathrm{GHz}$ observations were made with the $13.7 \mathrm{~m}$ diameter Metsähovi radio telescope, which is a radome enclosed paraboloid antenna situated in Finland (24 23' 38'E, +60 13' 05"). The measurements were made with a $1 \mathrm{GHz}$-band dual beam receiver centered at $36.8 \mathrm{GHz}$. The HEMPT (high electron mobility pseudomorphic transistor) front end operates at room temperature. The observations were taken with an $\mathrm{ON}-\mathrm{ON}$ technique, alternating the source and the sky in each feed horn. A typical integration time to obtain one flux density data point is between 1200 and $1400 \mathrm{~s}$. The detection limit of the telescope at $37 \mathrm{GHz}$ is on the order of $0.2 \mathrm{Jy}$ under optimal conditions. Data points with a signal-to-noise ratio $<4$ are treated as nondetections. The flux density scale is set by observations of thermal radio source DR 21. Sources NGC 7027, 3C 274, and 3C 84 are used as secondary calibrators. A detailed description of the data reduction and analysis is given in Teräsranta et al. (1998). The error estimate in the flux density includes the contribution from the measurement rms and the uncertainty of the absolute calibration. 


\subsection{OVRO Data}

As part of an ongoing blazar monitoring program, the Owens Valley Radio Observatory (OVRO) $40 \mathrm{~m}$ radio telescope has observed $4 \mathrm{C}+21.35$ at $15 \mathrm{GHz}$ regularly since the end of 2007 (Richards et al. 2011). This monitoring program includes about 1700 known or likely $\gamma$-ray-loud blazars, including all candidate $\gamma$-ray blazar survey (CGRaBS) sources above declination $-20^{\circ}$. The sources in this program are observed in total intensity twice per week with a $4 \mathrm{mJy}$ (minimum) and $3 \%$ (typical) uncertainty on the flux density. Observations are performed with a dual-beam (each 2.5 arcmin full-width half-maximum) Dickeswitched system using cold sky in the off-source beam as the reference. Additionally, the source is switched between beams to reduce atmospheric variations. The absolute flux density scale is calibrated using observations of $3 \mathrm{C} 286$, adopting the flux density (3.44 Jy) from Baars et al. (1977). This results in about a $5 \%$ absolute scale uncertainty, which is not reflected in the plotted errors. $4 \mathrm{C}+21.35$ was variable at $15 \mathrm{GHz}$ during the OVRO monitoring (Figure 2), with a flux density ranging from $1.01 \mathrm{Jy}$ (at MJD 55094) to 2.13 Jy (at MJD 55423).

\subsection{UMRAO Data}

UMRAO centimeter band total flux density observations were obtained with the University of Michigan $26 \mathrm{~m}$ paraboloid located in Dexter, Michigan, USA. The instrument is equipped with transistor-based radiometers operating at frequencies centered at 4.8, 8.0, and $14.5 \mathrm{GHz}$ with bandwidths of $0.68,0.79$, and $1.68 \mathrm{GHz}$, respectively. Dual horn feed systems are used at 8 and $14.5 \mathrm{GHz}$, while at $4.8 \mathrm{GHz}$ a single-horn, modeswitching receiver is employed. Each observation consisted of a series of 8-16 individual measurements over approximately a 25-45 minute time period, utilizing an on-off observing technique at $4.8 \mathrm{GHz}$, and an on-on technique (switching the target source between the two feed horns, which are closely spaced on the sky) at 8.0 and $14.5 \mathrm{GHz}$. As part of the observing procedure, drift scans were made across strong sources to verify the telescope pointing correction curves, and observations of nearby calibrators (3C 274, 3C 286, and 3C 218) were obtained every 1-2 hr to correct for temporal changes in the antenna aperture efficiency.

\subsection{Medicina Data}

We observed $4 \mathrm{C}+21.35$ with the Medicina radio telescope five times between 2010 April 26 and 2010 May 11. We used the new Enhanced Single-dish Control System (ESCS) acquisition system, which provides enhanced sensitivity and supports observations with the cross scan technique. We observed at $22 \mathrm{GHz}$ in the first four epochs, and at $5 \mathrm{GHz}$ in the last two; the observations on 2010 May 10 were carried out at both frequencies and can be used for an estimate of the simultaneous spectral index.

At each epoch, the source was observed for about 10 minutes and calibrated with respect to $3 \mathrm{C} 286$, after correcting the data for atmospheric opacity. The observing conditions varied from epoch to epoch, resulting in different noise levels and significance of the detections. However, after flagging bad scans, we always obtained a highly significant $(\gg 5 \sigma)$ detection. The relative uncertainty on the estimated flux density at $22 \mathrm{GHz}$ varies between $4 \%$ and $15 \%$, while at $5 \mathrm{GHz}$ it is around $3 \%$.

\subsection{MOJAVE Data}

$4 \mathrm{C}+21.35$ is part of the Monitoring of Jets in Active Galactic Nuclei with VLBA Experiments (MOJAVE) sample, allowing us to investigate at $15 \mathrm{GHz}$ the radio structure and jet kinematics of this source over a long period. The data were processed using the standard procedures described in the AIPS cookbook ${ }^{99}$ (for details, see Lister et al. 2009). The radio properties of $4 \mathrm{C}+21.35$ strongly indicate that it has a relativistic jet beamed very closely along our line of sight. The kiloparsec scale radio morphology from Very Large Array images (Cooper et al. 2007; Saikia et al. 1993) consists of a bright jet starting out to the northeast of a bright core, and gradually curving to the east, terminating in a hotspot located approximately $60 \mathrm{kpc}$ from the core. Surrounding the core is a circular halo of diffuse radio emission $\sim 100 \mathrm{kpc}$ in diameter, which is consistent with a large radio lobe being viewed end-on. On parsec scales, $4 \mathrm{C}+21.35$ displays a compact radio jet at an initial position angle of $\sim 0^{\circ}$ that curves roughly $7^{\circ}$ to the east over 10 mas. However, there is also a more distant feature at position angle $-6^{\circ}$ from the optically thick core. Multi-epoch Very Long Baseline Array (VLBA) observations by the MOJAVE survey, using data from 1996 until 2011 May (for details of the fitting method, see Lister et al. 2013) show that this outermost feature (id = 1) has an apparent superluminal motion of $8.4 c$, and is moving to the east (Figure 4). Several other jet features closer in have faster speeds, all close to $17 c$, and are also accelerating to the east. There are two components (ids $=6,7$ ) with even faster speeds of $20 c$ and $27 c$ (Table 3), that have trajectories curving to the west. These kinematic observations suggest complex three dimensional trajectories, perhaps having a helical form, which are being investigated in further detail by the MOJAVE collaboration. The linear fractional polarization and electric vector direction of the core feature changed between 2009 December and 2010 July, but remained relatively constant from 2010 July to December (Figure 5). There is evidence for a new bright jet feature in the core region as of 2009 November. The electric vector directions of the moving features further down the jet were remarkably uniform with time, pointing in a direction roughly perpendicular to the motion vector of the outermost moving features. On the other hand, there was no evidence at $15 \mathrm{GHz}$ of a bright superluminal knot ejection during the 2010 $\gamma$-ray flaring period.

\section{DISCUSSION}

\subsection{Light Curves Behavior and Correlation}

The multifrequency light curve of $4 \mathrm{C}+21.35$ in Figure 6 shows the Fermi-LAT, Swift (XRT; UVOT, $u$ and $m 2$ filters), optical $R$ band (Abastumani, ATOM, Crimean, KVA, St. Petersburg), and radio from $15 \mathrm{GHz}$ to $37 \mathrm{GHz}$ (Effelsberg, Medicina, Metsähovi, OVRO, UMRAO) data collected during 2010 April 9-August 4 (MJD 55295-55412). In addition the $\gamma$-ray and X-ray photon indices observed by Fermi-LAT and Swift-XRT are reported in the second and fourth panels. The Fermi-LAT light curve shows two distinct $\gamma$-ray flaring episodes, peaking on 2010 April 29 (MJD 55315) and June 18 (MJD 55365), together with other peaks of lesser brightness. The two major $\gamma$-ray peaks detected by Fermi-LAT occurred very close in time to the VHE detections by MAGIC, on May 3 and June 17. This indicates that the same emission mechanism may be responsible for both the HE and VHE emission during these flaring episodes, in agreement also with the fact that the combined HE and VHE spectrum in 2010 June 17, corrected for the EBL absorption, can be described by a single power-law

\footnotetext{
99 http://www.aips.nrao.edu
} 


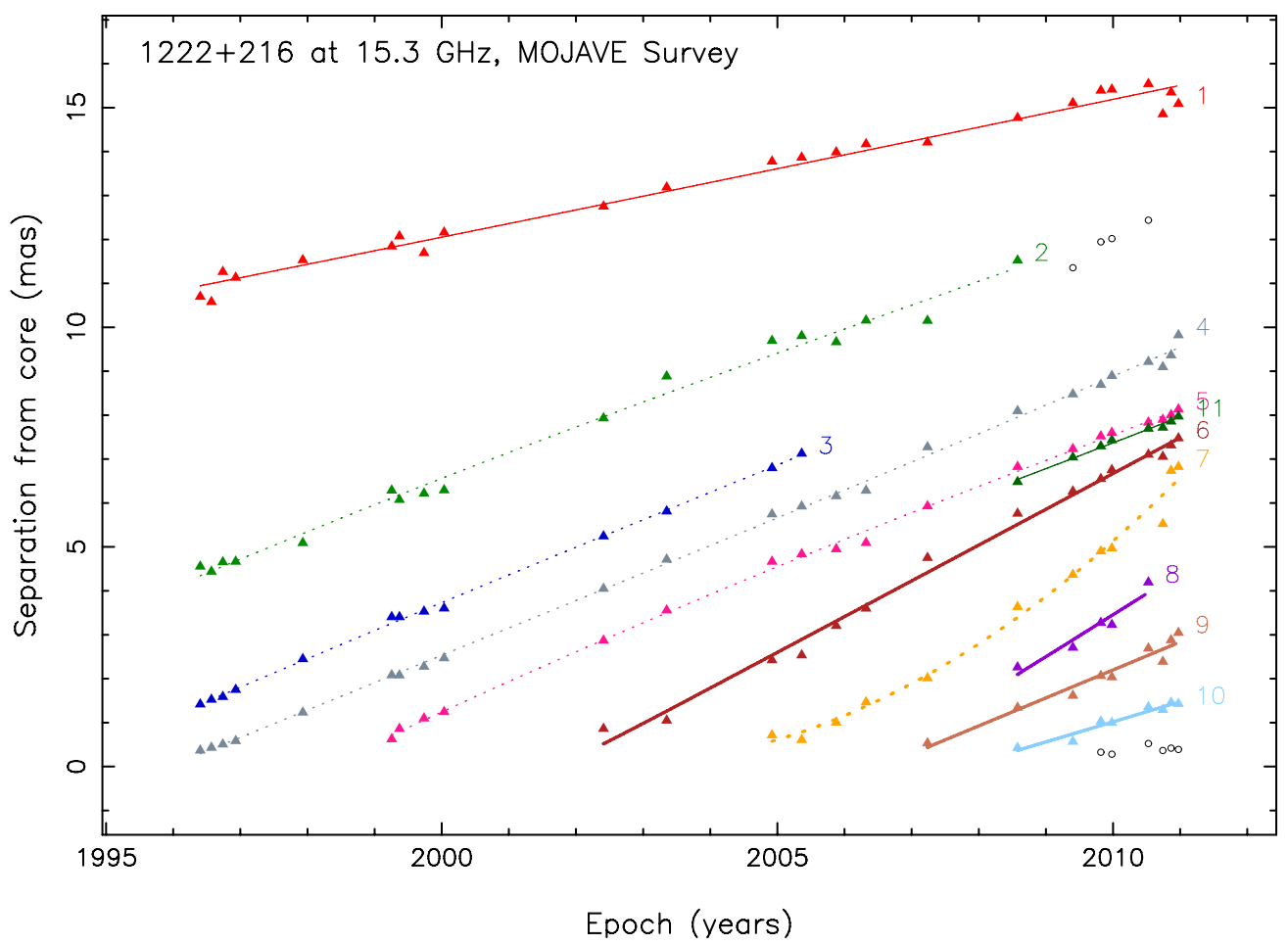

Figure 4. Plot of angular separation from core vs. epoch for fitted Gaussian jet components in $4 \mathrm{C}+21.35$. Color symbols indicate robust components for which kinematic fits were obtained (dotted and solid lines). The solid lines indicate vector motion fits to the data points assuming no acceleration, while the dotted lines indicate accelerated motion fits. Thick lines are used for components whose fitted motion is along a radial direction from the core, while the thin lines indicate non-radial motions. Unfilled black circles indicate non-robust components. The component identification numbers are located next to the last epoch of each robust component.

(A color version of this figure is available in the online journal.)

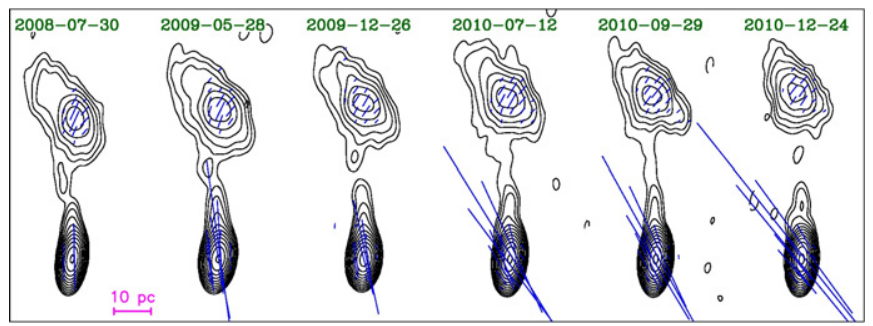

Figure 5. Total intensity and linear polarization images of $4 C+21.35$ observed by VLBA at $15 \mathrm{GHz}$ in different epochs as part of the MOJAVE program. Naturally weighted total intensity images are shown by black contours, the contours are in successive powers of two times the base contour level of $0.2 \mathrm{mJy}$ beam $^{-1}$. Electric polarization vectors direction is indicated by blue sticks, their length is proportional to the polarized intensity.

(A color version of this figure is available in the online journal.)

(Aleksić et al. 2011). It is also worth noting that during the two VHE detections the photon index estimated in the LAT energy range is quite flat $\left(\Gamma_{\gamma} \sim 2\right)$, favoring the detection of $\gamma$-ray emission up to hundreds of $\mathrm{GeV}$.

During 2010 February-June, Swift /XRT observed 4C +21.35 with a $0.3-10 \mathrm{keV}$ flux in the range (2.6-7.7) $\times$ $10^{-12} \mathrm{erg} \mathrm{cm}^{-2} \mathrm{~s}^{-1}$, with the photon index changing in the range 1.4-2.2. The photon index remained constant during the 2010 April and June $\gamma$-ray flaring periods (see Figure 6). The very small variability amplitude observed in X-rays $(\sim 3)$ with respect to the $\mathrm{MeV}-\mathrm{GeV}$ energy range $(\sim 70)$ could indicate that the low-energy segment of the electron energy distribution responsible for the production of the X-ray photons varies much less than the high-energy electron tail involved in the production of the observed $\gamma$-ray emission. A small variability amplitude was observed in UV during 2010. This could be due to the fact that the UV part of the spectrum is dominated by the accretion disk emission that dilutes the jet emission. It is worth noting that a peak of the UV emission was detected on June 18, but the small increase observed makes it unlikely that the change of the accretion rate is the main driver of the simultaneous activity observed at the higher energies by MAGIC and Fermi-LAT.

The $R$-band light curve is quite well sampled and shows variable flux density over time, but no dramatic increase of the activity. Two optical peaks were observed on 2010 April 20 (MJD 55306) and 2010 June 30 (MJD 55377), close in time but not simultaneous with the two $\gamma$-ray peaks. For the second flaring event, the lack of strictly simultaneous ground-based optical observations was covered by the UVOT observations that seems to indicate a relatively high activity at MJD 55367 (June 20). Correlations between the $\gamma$-ray and optical light curves of $4 \mathrm{C}+21.35$ were investigated by computing the discrete cross correlation function (DCCF), following Edelson and Krolik (1988) and White and Peterson (1994; see Figure 7; positive lag means that $\gamma$-ray flux variations occur before those in $R$ band; the DCCF value ranges from -1 to +1 ). Although the overall $R$-band flux was higher during the period of $\gamma$-ray activity (see Figure 8 ) the DCCF shows no clear evidence for correlations on the timescale of the rapid flares ( $\sim$ days), with a maximum correlation of 0.4 for a time-lag of $\sim 35$ days. A similar conclusion was reached by Smith et al. (2011) from a comparison of a LAT light curve during this epoch with the Steward Observatory $V$-band observations also used in this paper. In particular, overall correlation between the $\gamma$-ray band with the $R$ band was observed for 4C 21+35 during the 2010 $\gamma$-ray flaring activity, but on short time scales some differences 
Table 3

Kinematic Fit Properties of Jet Components

\begin{tabular}{|c|c|c|c|c|c|c|c|c|c|c|c|c|c|c|}
\hline $\begin{array}{l}\text { I.D. } \\
\text { (1) }\end{array}$ & $\begin{array}{c}N \\
(2)\end{array}$ & $\begin{array}{c}\langle S\rangle \\
(\mathrm{mJy}) \\
(3)\end{array}$ & $\begin{array}{c}\langle R\rangle \\
(\mathrm{mas}) \\
(4)\end{array}$ & $\begin{array}{c}\langle\vartheta\rangle \\
(\operatorname{deg}) \\
(5)\end{array}$ & $\begin{array}{c}\phi \\
(\mathrm{deg}) \\
(6)\end{array}$ & $\begin{array}{c}|\langle\vartheta\rangle-\phi| \\
\text { (deg) } \\
\text { (7) }\end{array}$ & $\begin{array}{c}\mu \\
\left(\mu \text { as yr}^{-1}\right) \\
(8)\end{array}$ & $\begin{array}{l}\beta_{\text {app }} \\
(c) \\
(9)\end{array}$ & $\begin{array}{c}\dot{\mu}_{\perp} \\
\left(\mu \mathrm{as} \mathrm{yr}^{-2}\right) \\
(10)\end{array}$ & $\begin{array}{c}\dot{\mu}_{\|} \\
\left(\mu \mathrm{as}^{-2}\right) \\
(11)\end{array}$ & $\begin{array}{c}T_{e j} \\
(12)\end{array}$ & $\begin{array}{l}T_{\text {mid }} \\
(13)\end{array}$ & $\begin{array}{c}\Delta \alpha \\
(\mu \text { as }) \\
(14)\end{array}$ & $\begin{array}{c}\Delta \delta \\
(\mu \mathrm{as}) \\
(15)\end{array}$ \\
\hline 1 & 24 & 14 & 13.5 & 354.1 & $11.5 \pm 1.4$ & $17.4 \pm 1.4^{\mathrm{b}}$ & $320.4 \pm 9.3$ & $8.41 \pm 0.24$ & $0.049 \pm 0.017$ & $-0.038 \pm 0.021$ & $\ldots$ & 2003.84 & 199 & 245 \\
\hline 2 & 17 & 20 & 7.4 & 7.4 & $15.7 \pm 1.2$ & $8.2 \pm 1.2^{\mathrm{b}}$ & $581 \pm 17^{\mathrm{a}}$ & $15.26 \pm 0.43$ & $0.092 \pm 0.019$ & $-0.007 \pm 0.026$ & $\ldots$ & 2002.49 & 179 & 269 \\
\hline 3 & 13 & 74 & 3.7 & 2.7 & $10.19 \pm 0.27$ & $7.48 \pm 0.30^{\mathrm{b}}$ & $640.6 \pm 8.4^{\mathrm{a}}$ & $16.82 \pm 0.22$ & $0.0730 \pm 0.0055$ & $0.005 \pm 0.015$ & $\ldots$ & 2000.88 & 29 & 93 \\
\hline 4 & 25 & 101 & 5.3 & 4.3 & $9.71 \pm 0.39$ & $5.41 \pm 0.46^{\mathrm{b}}$ & $638.9 \pm 5.1^{\mathrm{a}}$ & $16.77 \pm 0.13$ & $0.0576 \pm 0.0053$ & $0.0094 \pm 0.0063$ & $\ldots$ & 2003.84 & 114 & 136 \\
\hline 5 & 20 & 71 & 5.2 & 359.5 & $5.73 \pm 0.21$ & $6.22 \pm 0.23^{b}$ & $631.2 \pm 5.8^{\mathrm{a}}$ & $16.57 \pm 0.15$ & $0.0743 \pm 0.0034$ & $-0.0225 \pm 0.0082$ & $\ldots$ & 2005.26 & 43 & 109 \\
\hline 6 & 16 & 24 & 5.1 & 355.8 & $353.19 \pm 0.99$ & $2.6 \pm 1.1$ & $779 \pm 15$ & $20.45 \pm 0.40$ & $-0.040 \pm 0.020$ & $0.012 \pm 0.025$ & $2001.53 \pm 0.14$ & 2006.84 & 153 & 171 \\
\hline 7 & 14 & 19 & 4.1 & 4.5 & $2.96 \pm 0.94$ & $1.5 \pm 1.1$ & $1013 \pm 39^{\mathrm{a}}$ & $26.6 \pm 1.0$ & $-0.105 \pm 0.033$ & $0.312 \pm 0.078$ & $\ldots$ & 2008.10 & 136 & 323 \\
\hline 9 & 10 & 8 & 2.1 & 0.9 & $0.16 \pm 0.87$ & $0.71 \pm 0.92$ & $632 \pm 45$ & $16.6 \pm 1.2$ & $0.012 \pm 0.038$ & $0.23 \pm 0.15$ & $2006.55 \pm 0.25$ & 2009.26 & 36 & 168 \\
\hline 10 & 9 & 26 & 1.1 & 4.3 & $6.9 \pm 2.6$ & $2.6 \pm 2.7$ & $444 \pm 35$ & $11.67 \pm 0.92$ & $\ldots$ & $\ldots$ & $2007.82 \pm 0.20$ & 2009.93 & 49 & 87 \\
\hline 11 & 9 & 27 & 7.5 & 357.8 & $17.7 \pm 2.3$ & $19.9 \pm 2.3^{b}$ & $615 \pm 23$ & $16.15 \pm 0.60$ & $\ldots$ & $\ldots$ & $\ldots$ & 2009.93 & 61 & 56 \\
\hline 12 & 7 & 99 & 0.4 & 8.4 & $18.7 \pm 4.5$ & $10.3 \pm 4.5$ & $96 \pm 19$ & $2.52 \pm 0.49$ & $\ldots$ & $\ldots$ & $\ldots$ & 2010.55 & 6 & 26 \\
\hline
\end{tabular}

Notes. The kinematic fit values are derived from the acceleration fit for components with significant acceleration, and from the vector motion fit otherwise. Columns are as follows: (1) component number, (2) number of fitted epochs, (3) mean flux density at $15 \mathrm{GHz}$ in mJy, (4) mean distance from core component in mas, (5) mean position angle with respect to the core component in degrees, (6) position angle of velocity vector in degrees, (7) offset between mean position angle and velocity vector position angle in degrees, (8) angular proper motion in microarcseconds per year, (9) fitted speed in units of the speed of light, (10) angular acceleration perpendicular to velocity direction in microarcseconds per year per year, (11) angular acceleration parallel to velocity direction in microarcseconds per year per year, (12) fitted ejection date, (13) date of reference (middle) epoch used for fit, (14) right ascension error of individual epoch positions in $\mu$ as, (15) declination error of individual epoch positions in $\mu$ as.

${ }^{\text {a }}$ Component shows significant accelerated motion.

${ }^{\mathrm{b}}$ Component shows significant non-radial motion. 


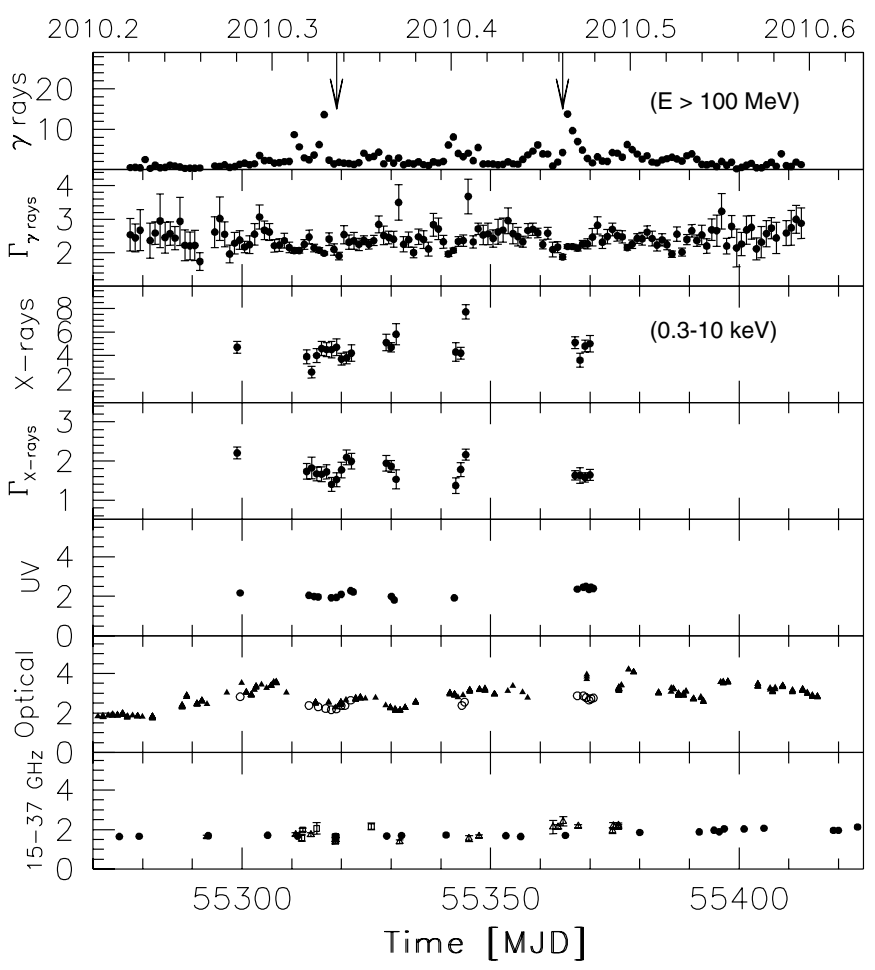

Figure 6. Multifrequency light curves of $4 \mathrm{C}+21.35$ between 2010 April 9 and August 4 (MJD 55295-55412). The data sets were collected (from top to bottom) by Fermi-LAT ( $E>100 \mathrm{MeV}$; in units of $10^{-6}$ photons $\mathrm{cm}^{-2} \mathrm{~s}^{-1}$; taken from Tanaka et al. 2011), Swift-XRT (0.3-10 keV; in units of $\left.10^{-12} \mathrm{erg} \mathrm{cm}^{-2} \mathrm{~s}^{-1}\right)$, Swift-UVOT (m2 filter; in units of mJy), Swift-UVOT (U filter, open circles; in units of mJy), Abastumani, ATOM, Crimean, KVA, St. Petersburg ( $R$-band, filled triangles; in units of mJy), Effelsberg, Medicina, Metsähovi, OVRO, UMRAO (15 GHz: filled circles, $23 \mathrm{GHz}$ : open squares, $37 \mathrm{GHz}$ : open triangles; in units of Jy). The downward arrows indicate the times of the VHE detections by MAGIC. For clarity the $m 2, u, R$ and $15 \mathrm{GHz}$ bands errors (typically $5 \%$ or less) and the $\gamma$-rays errors are not shown.

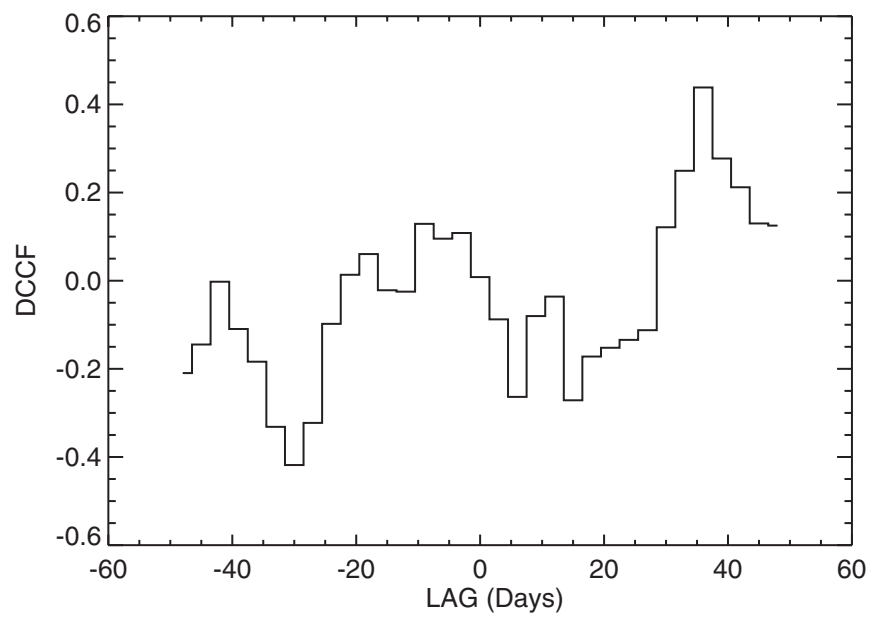

Figure 7. Discrete cross correlation function between the $\gamma$-ray and $R$-band light curves of $4 \mathrm{C}+21.35$.

are evident (see Figure 8). A complex connection between the optical and $\gamma$-ray emission has been already observed in several FSRQs and low-synchrotron-peaked BL Lacertae objects. In some cases a clear optical $/ \gamma$ correlation with no lags was observed (e.g., 3C 279; Abdo et al. 2010). But sometimes no correlation was found between these two energy bands (e.g., BL Lac; Abdo et al. 2011), and in other occasions, an optical and NIR flare with no significant counterpart in $\gamma$-rays was ob-

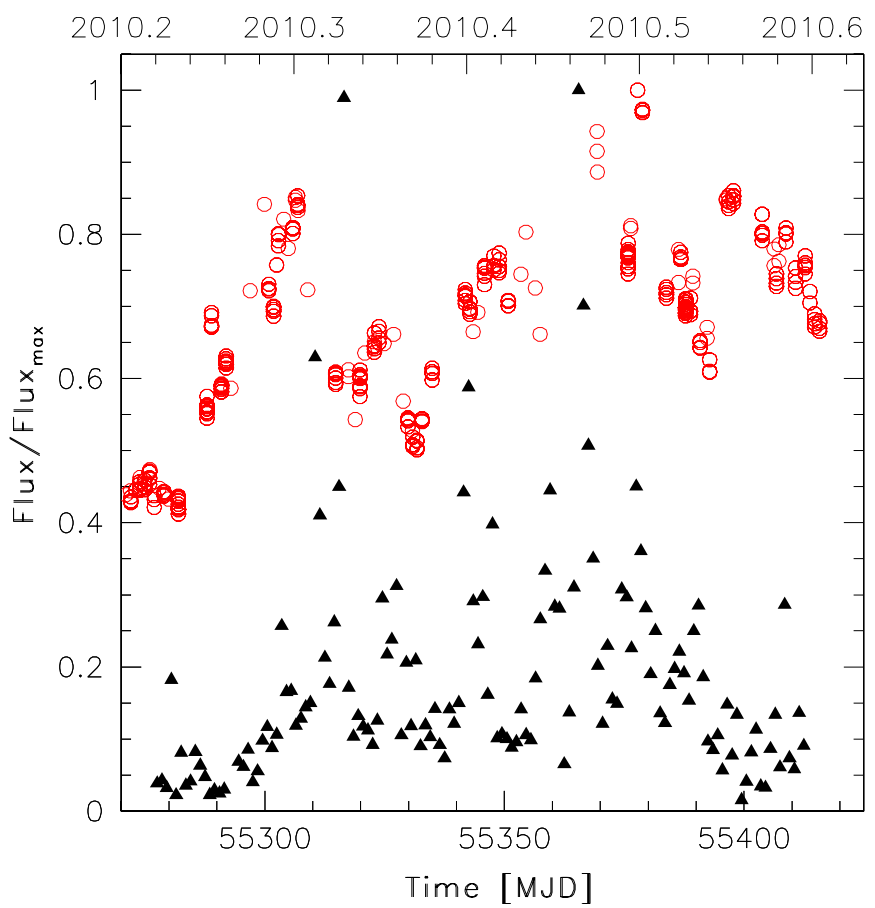

Figure 8. Comparison between $\gamma$-ray and $R$-band light curves. We superimpose $\gamma$-ray (black triangles) and $R$-band (red empty circles) light curves normalizing $\gamma$-ray and $R$ flux values over the whole observing period to the respective peak flux values.

(A color version of this figure is available in the online journal.)

served (e.g., PKS 0208-512 and PKS 0537-441; Chatterjee et al. 2013; D'Ammando et al. 2013).

An increasing flux density was observed in radio and $\mathrm{mm}$ bands from the beginning of 2009 (see Figure 2) contemporaneous with the increasing $\gamma$-ray activity observed by Fermi-LAT, reaching the peak of flux density at $230 \mathrm{GHz}$ on 2011 January 27 (MJD 55588). Interestingly, the peak of the $23 \mathrm{GHz}$ and $37 \mathrm{GHz}$ was observed on 2010 May 10 (MJD 55326) and June 18 (MJD 55365), respectively, close in time with the major $\gamma$-ray flares. The same activity was also observed at $8 \mathrm{GHz}$ and $5 \mathrm{GHz}$, with the emission peak delayed likely due to synchrotron self-absorption opacity effects. However, the sparse coverage does not allow us to obtain conclusive evidence. A significant spectral evolution was also observed in radio (see Figure 3), with the spectrum changed from steep on 2009 January $24\left(\alpha_{r}=0.3\right)$ to inverted $\left(\alpha_{r}=-0.2\right)$ on 2011 April 29 (see Section 6.2).

\subsection{SED Modeling \\ 7.2.1. Data Selection}

We have built three quasi-simultaneous SEDs from the data discussed above, shown in Figure 9. These SEDs include the flaring states of 2010 June 17 (red circles) and 2010 April 29 (green squares), and a quiescent state, integrated in time from 2008 August 4 to 2008 September 12 (blue diamonds). For the three SEDs we used the LAT spectra calculated over 2010 June 17, 2010 April 23-May 2, 2008 August 4-2009 September 12 (taken from Tanaka et al. 2011; Aleksić et al. 2011), and the Swift data collected on 2010 June 20, 2010 April 23, and 2009 April 10, respectively. The MAGIC data collected on 2010 May 3 and June 17 are included for the two flaring states. These data have been corrected for EBL absorption using the model of Finke et al. (2010). This model is 


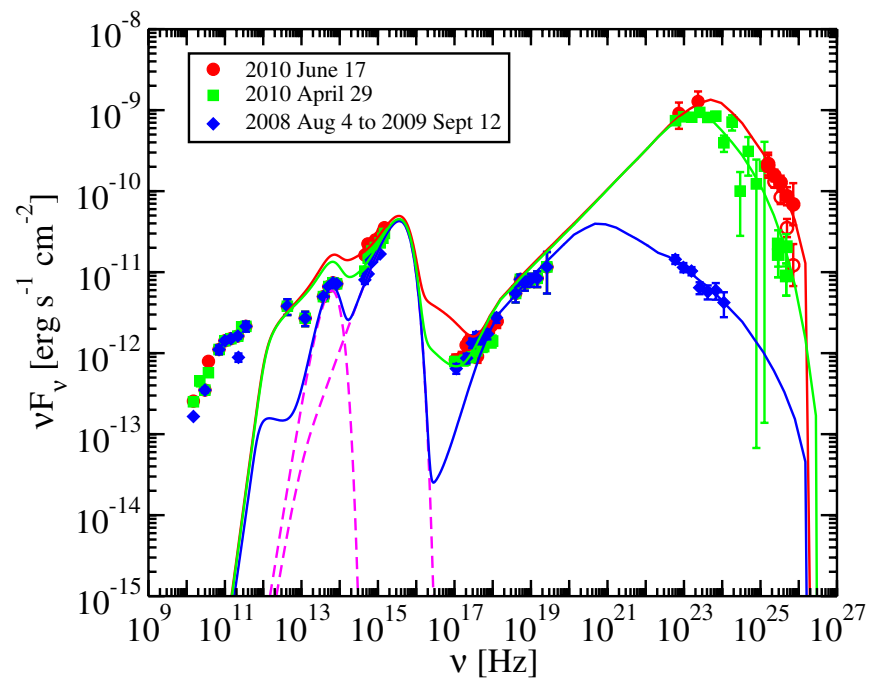

Figure 9. Spectral energy distribution of $4 \mathrm{C}+21.35$ in three epochs: 2010 June 17 (red circles), 2010 April 29 (green squares), and 2008 August 4-2009 September 12 (blue diamonds). Dashed magenta lines indicate the dust torus and accretion disk emission components. The MAGIC data have been corrected for EBL absorption using the model of Finke et al. (2010). Empty symbols refer to non-EBL-corrected data, filled symbols to EBL-corrected ones.

(A color version of this figure is available in the online journal.)

nearly identical in the energy range covered by MAGIC to the model of Dominguez et al. (2011) used in Aleksić et al. (2011). We also included the radio and $R$-band observations nearest to the LAT $\gamma$-ray peak for the two flaring states (2010 April 28 and June 22, respectively), and the Swift observation performed on 2009 April 19 for the quiescent state. Finally we report in the SEDs the average Swift BAT 70 month spectrum, the Planck spectrum collected in 2009 December (Ade et al. 2013), and the Spitzer data from Malmrose et al. (2011).

Farina et al. (2012) estimate the black hole (BH) mass for $4 \mathrm{C}+21.35$ as $M_{\mathrm{BH}} \sim 6 \times 10^{8} M_{\odot}$, using broad emission line measurements from over 100 optical spectra from a variety of sources. This value agrees with values found by Shen et al. (2011) and Shaw et al. (2012) with an Sloan Digital Sky Survey (SDSS) spectrum, but significantly greater than the value found by Wang et al. (2004) and used by Tanaka et al. (2011), $M_{\mathrm{BH}} \sim 1.5 \times 10^{8} M_{\odot}$. It is worth noting that the measurement by Wang et al. (2004) relies on the $\mathrm{H}-\beta$ broad line and the continuum luminosity-BLR radius relation from Kaspi et al. (2000). That relation was obtained from reverberation mapping of a small number of active galactic nuclei using a cosmology with a decelerating universe, which is now known to be incorrect. We use the more precise value from Farina et al. (2012) in our SED modeling.

\subsubsection{Model}

We model the SED of the three epochs using a one-zone leptonic model. We began our modeling efforts by attempting to model the IR data from Malmrose et al. (2011) with a blackbody dust torus. The results for the luminosity $\left(L_{\text {dust }}\right)$ and temperature $\left(T_{\text {dust }}\right)$ of the blackbody were similar to the ones found by Malmrose et al. (2011). The optical emission clearly appears to be dominated by thermal disk emission, rather than nonthermal synchrotron emission from the jet, otherwise the optical spectrum would appear much softer. Therefore, we next modeled the optical data in the low-state with a Shakura-Sunyaev multi-temperature disk (Shakura \& Sunyaev 1973), assuming $M_{\mathrm{BH}} \sim 6 \times 10^{8} M_{\odot}$. We note that the disk

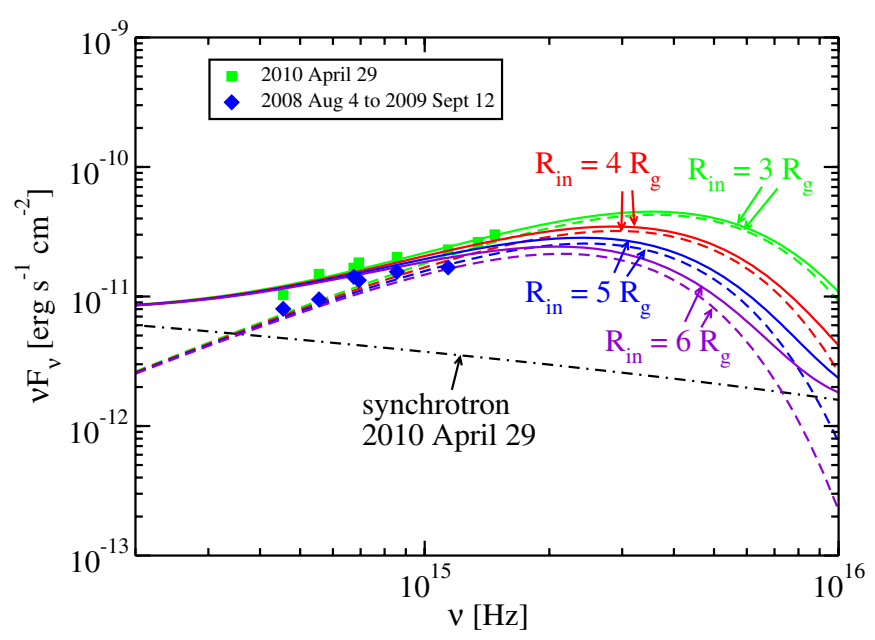

Figure 10. Similar to Figure 9, but zoomed in on the optical portion of the spectrum, which in our model originates mainly from disk emission. Model disk emission for several inner disk radii are shown (dashed curves), while the synchrotron from the model fit of 2010 April 29 is shown as the dot-dashed curve. The total (synchrotron + disk) emission is shown as the solid curves. Models with large inner disk radii do not provide an adequate fit to the UV data of 2010 April 29.

(A color version of this figure is available in the online journal.)

fit to the low-state data is insensitive to the inner radius of the disk, $R_{i n}$, as seen in a close up of this part of the SED in Figure 10. Parameters for the dust torus, accretion disk, and all other modeling parameters can be found in Table 4. Tavecchio et al. (2011) use an isotropically emitting blackbody spectrum to fit the blue bump and obtain a value for the disk luminosity over twice the value presented here. We use a Shakura-Sunyaev disk, which does not emit isotropically, and which we assume emits as the cosine of the disk inclination angle. With this distribution, for a face-on disk, the flux will be twice that from an isotropic distribution for a given luminosity (e.g., Castignani et al. 2013). This is the cause of most of the discrepancy, with the remaining discrepancy due to the different contributions from nonthermal synchrotron emission.

Although several possibilities have been suggested for the origin of $\gamma$-ray emission from $4 \mathrm{C}+21.35$ (see Section 1), FSRQ-type blazars such as $4 \mathrm{C}+21.35$ are expected to have their $\gamma$-rays originate from the external Compton (EC), rather than synchrotron self-Compton (SSC) mechanism (e.g., Ghisellini et al. 1998). Therefore we next attempt to fit the SED in the high state of 2010 June 17 (MJD 55364) with a combination of synchrotron, SSC, and EC emission from a jet blob moving at a highly relativistic speed. We assume an emitting size of $R_{b}^{\prime}=10^{15} \mathrm{~cm}$ in the comoving frame, consistent with the rapid variability timescale of 10 minutes. The dust torus and disk emission are not varied between flaring and quiescent states. For the nonthermal jet emission we choose a variability timescale of 10 minutes, consistent with the variability observed by MAGIC (Aleksic et al. 2011). The MAGIC detection of the source out to $\gtrsim 300 \mathrm{GeV}$ also implies the primary emitting region must be outside the BLR, otherwise $\gamma \gamma$ absorption by broad-line photons would not allow such high-energy $\gamma$-ray photons to escape (Tanaka et al. 2011; Aleksić et al. 2011), so we chose a large jet distance from the $\mathrm{BH}, r$, outside the BLR radius of $R_{\mathrm{BLR}} \approx 2 \times 10^{17} \mathrm{~cm}$ (Tanaka et al. 2011). Outside the BLR, the seed photon source is expected to be from the dust torus, which is what we use as the EC seed photon source. For the purposes of calculating the geometry of Compton scattering, 
Table 4

Model Parameters for the SED Shown in Figure 9

\begin{tabular}{|c|c|c|c|c|}
\hline Parameter & Symbol & 2010 June 17 & 2010 April 29 & Quiescent State \\
\hline Gravitational radius $(\mathrm{cm})$ & $R_{g}$ & $8.8 \times 10^{13}$ & $8.8 \times 10^{13}$ & $8.8 \times 10^{13}$ \\
\hline Eddington luminosity $\left(\mathrm{erg} \mathrm{s}^{-1}\right)$ & $L_{\text {Edd }}$ & $7.8 \times 10^{46}$ & $7.8 \times 10^{46}$ & $7.8 \times 10^{46}$ \\
\hline Disk Eddington ratio & $L_{\text {disk }} / L_{\text {Edd }}$ & 0.2 & 0.2 & 0.2 \\
\hline Disk accretion efficiency & $\eta_{\text {disk }}$ & $1 / 12$ & $1 / 12$ & $1 / 12$ \\
\hline Inner disk radius $\left(R_{g}\right)$ & $R_{\text {in }}$ & 3 & 3 & 3 \\
\hline Outer disk radius $\left(R_{g}\right)$ & $R_{\text {out }}$ & $3 \times 10^{4}$ & $3 \times 10^{4}$ & $3 \times 10^{4}$ \\
\hline Bulk Lorentz factor & $\Gamma$ & 40 & 40 & 40 \\
\hline Doppler factor & $\delta_{\mathrm{D}}$ & 80 & 80 & 80 \\
\hline Magnetic field & $B(\mathrm{G})$ & 0.7 & 0.7 & 0.7 \\
\hline Variability timescale & $t_{v}(\mathrm{~s})$ & $6 \times 10^{2}$ & $6 \times 10^{2}$ & $6 \times 10^{2}$ \\
\hline Comoving radius of blob & $R_{b}^{\prime}(\mathrm{cm})$ & $1.0 \times 10^{15}$ & $1.0 \times 10^{15}$ & $1.0 \times 10^{15}$ \\
\hline Jet height $(\mathrm{cm})$ & $r$ & $8.8 \times 10^{18}$ & $8.8 \times 10^{18}$ & $8.8 \times 10^{18}$ \\
\hline Low-energy electron spectral index & $p_{1}$ & 2.0 & 2.0 & 2.0 \\
\hline High-energy electron spectral index & $p_{2}$ & 3.5 & 3.5 & 3.5 \\
\hline Minimum electron Lorentz factor & $\gamma_{\min }^{\prime}$ & 1.0 & 1.0 & 1.0 \\
\hline Break electron Lorentz factor & $\gamma_{\text {brk }}^{\prime}$ & $1.0 \times 10^{3}$ & $6.0 \times 10^{2}$ & 26 \\
\hline Maximum electron Lorentz factor & $\gamma_{\max }^{\prime}$ & $4.0 \times 10^{4}$ & $2.0 \times 10^{4}$ & $4.0 \times 10^{4}$ \\
\hline Dust torus luminosity $\left(\mathrm{erg} \mathrm{s}^{-1}\right)$ & $L_{\text {dust }}$ & $5.5 \times 10^{45}$ & $5.5 \times 10^{45}$ & $5.5 \times 10^{45}$ \\
\hline Dust torus temperature $(\mathrm{K})$ & $T_{\text {dust }}$ & $1.1 \times 10^{3}$ & $1.1 \times 10^{3}$ & $1.1 \times 10^{3}$ \\
\hline Dust torus radius $(\mathrm{cm})$ & $R_{\text {dust }}$ & $1.8 \times 10^{19}$ & $1.8 \times 10^{19}$ & $1.8 \times 10^{19}$ \\
\hline Jet power in magnetic field (erg s $\left.{ }^{-1}\right)$ & $P_{j, B}$ & $5.9 \times 10^{42}$ & $5.9 \times 10^{42}$ & $5.9 \times 10^{42}$ \\
\hline Jet power in electrons $\left(\mathrm{erg} \mathrm{s}^{-1}\right)$ & $P_{j, e}$ & $4.3 \times 10^{44}$ & $4.0 \times 10^{44}$ & $1.9 \times 10^{44}$ \\
\hline
\end{tabular}

Note. A black hole mass of $6 \times 10^{8} M_{\odot}$ was considered.

we assume the dust torus is a one-dimensional ring with radius $R_{\text {dust }}$, aligned orthogonal to the jet, where we choose $R_{\text {dust }}$ to be roughly consistent with the value of the dust sublimation radius calculated by Nenkova et al. (2008). This is necessary since our calculations use the full angle-dependent Compton cross section, accurate in the Thomson through Klein-Nishina (KN) regimes. The adopted synchrotron component is self-absorbed below $10^{12} \mathrm{~Hz}$. We treat the radio points as upper limits, since their slow variability compared to the optical and $\gamma$-ray emission and flat spectrum (in flux density $F_{\nu}$ ) imply they are probably the result of a superposition of several self-absorbed jet components (Konigl 1981), and not the result of the same emitting region that produces the rest of the SED. The electron distribution was assumed to be a broken power law between electron Lorentz factors $\gamma_{\min }$ and $\gamma_{\max }$ with power-law index $p_{1}$ for $\gamma<\gamma_{\text {brk }}$ and $p_{2}$ for $\gamma>\gamma_{\text {brk }}$. Further details on the model and its parameters can be found in Finke et al. (2008) and Dermer et al. (2009).

The result of this fit to the 2010 June 17 (MJD 55364) SED is shown in Figure 9. We note that there is some degeneracy in the choice of the model parameters, hence the set of parameter values describing the observational data are not unique. However, we do demonstrate that a one-zone model can adequately describe the data. To account for the highest speeds derived by the jet kinematics analysis of the MOJAVE data (see Section 6.7) at least some portion of the jet must be viewed within $\sim 4^{\circ}$ of the line of sight. To avoid the extreme KN regime for Compton scattering, we found that the jet needs to be highly aligned, with the jet angle with respect to the line of sight $\theta \approx 0^{\circ}\left(\delta_{D} \approx 2 \Gamma\right)$, where $\delta_{D}$ is the Doppler factor. This is because the energy at which the extreme $\mathrm{KN}$ regime begins is at $\epsilon_{\mathrm{KN}} \approx\left(\delta_{D} / \Gamma\right) \epsilon_{0}$, where $\epsilon_{0}$ is the seed photon energy. Such a small jet's angle does not disagree with the high apparent speeds estimated on the scales of a few parsecs if the complex three dimensional trajectories observed by MOJAVE are taken into consideration. In fact, there is evidence for a bend in the jet on the parsec scale in the VLBA images, where the emission in this model originates (see Section 6.7). The model does not provide a good fit to the XRT data in this SED, with the model being dominated by synchrotron emission for the soft X-rays, while the XRT spectral index is $\Gamma_{\mathrm{X}}<2$ indicating it is dominated by some sort of Compton scattering, either SSC or EC (EC in the case of our model fit). However, the XRT data were not strictly simultaneous with the rest of the SED, particularly the LAT data (with a gap of six and three days between the X-ray and $\gamma$-ray data, respectively). As can be seen in Figure 6, the XRT photon index alternates between $\Gamma_{\mathrm{X}}<2$, implying Compton scattering dominates in this waveband, and $\Gamma_{X}>2$, implying synchrotron dominates. If the primary emitting region makes up the majority of the jet cross section, this model fit gives a jet half-opening angle of $\theta_{\text {open }} \sim R_{b}^{\prime} / r \sim 10^{-4} \mathrm{rad} \sim 0.007$, where $R_{b}^{\prime}$ is the comoving radius of the blob. Such a small opening angle is highly unlikely and inconsistent with radio observations (Figure 5), so this model implies that the overwhelming majority of the source's emission is coming from a very small fraction of the jet's cross section. We also calculated the jet power in electrons $\left(P_{j, e}\right)$ and Poynting flux $\left(P_{j, B}\right)$ for this model fit, assuming a two-sided jet (Finke et al. 2008), finding that the source has almost 100 times as much power in electrons as in Poynting flux. The model fit to the 2010 June 17 (MJD 55364) flaring SED is similar to the "case A" fit to the same SED data by Tavecchio et al. (2011). They also found a jet where the electron energy density dominates over the magnetic energy density, although in their case it is even more dominant, with $P_{j, e} \sim 10^{4} P_{j, B}$. Tavecchio et al. (2011) also provide two other fits to the same SED with two zone models: a "case B" where there is an additional contribution from a larger blob outside the BLR; and a "case C" where there is a contribution from a larger blob inside the BLR. Neither of these two-zone fits solves the problem of having an extremely small, bright blob at a large distance from the $\mathrm{BH}$, although they do provide fits much closer to equipartition between electrons and Poynting flux. The UV data for the 2010 June 17 flaring SED requires an inner disk 
radius $R_{i n}<6 R_{g}$, with the best fit found for $R_{i n}=3 R_{g}\left(R_{g}\right.$ is the gravitational radius). We discuss the implications of this below.

First, however, we discuss the fit to the other bright flare, on 2010 April 29. This SED is quite similar to the 2010 June 17 one, and we found we could fit this SED with only minor changes in the electron distribution, keeping the other parameters the same. Specifically, this required lowering $\gamma_{\text {brk }}$ from $1 \times 10^{3}$ to $6 \times 10^{2}$ and $\gamma_{\max }$ from $4 \times 10^{4}$ to $2 \times 10^{4}$. This resulted in a slightly lower $P_{j, e}$, as seen in Table 4 . For this flaring state, the lower $\gamma_{\max }$ yields a better fit for the XRT data. The UV data for this state also are more consistent with an inner disk radius $R_{\text {in }}=3 R_{g}$.

Finally, we turn to the "quiescent state" SED, derived by integrating LAT data from 2008 August 4 to 2009 September 12 in addition to multifrequency data in the same period. We again find a good fit changing only the electron distribution parameters from the flaring states, while keeping the rest of the parameters the same. Here we varied the electron break to $\gamma_{\text {brk }}=26$, and changed the normalization, keeping all other parameters the same as the fit to the 2010 June 17 flaring state. This provides a good fit to the SED, although it presents some peculiarities. In this model, the synchrotron peak would be observed at frequency $v_{p k} \approx m_{e} c^{2} / h \gamma_{\text {brk }}^{2} B / B_{\text {crit }} \delta_{D} /(1+z) \approx 7.4 \times 10^{10} \mathrm{~Hz}$, if this part of the spectrum is not highly synchrotron self-absorbed. Instead the peak is at $\sim 10^{12} \mathrm{~Hz}$, at the self-absorption frequency, where the model flux is about an order of magnitude below the data. This is not strictly a problem, since the observed radio emission is probably from a much larger region of the jet, but it does seem strange to have such a low synchrotron peak frequency. For the fit to the quiescent state, the model underpredicts the softest XRT flux, rather than overpredicting it as the model for the 2010 June 17 flare did. Again, this could be due to variability during this rather long quiescent time period. It is also possible that the $\mathrm{X}$-ray emission originates from a different region, maybe even from an accretion disk corona, particularly since the accretion disk is so prominent. There have been many instances in FSRQs where the X-ray continuum has been characterized by very distinct variability properties compared to optical and $\gamma$-ray flares (e.g., Abdo et al. 2010; Marscher et al. 2010).

\subsubsection{Accretion Disk Emission}

For both the flaring state models, we find that a good fit to the UV data from Swift-UVOT requires an inner disk radius $R_{i n}<$ $6 R_{g}$, where $6 R_{g}$ is the value one would expect for the innermost stable circular orbit around a nonrotating Schwarzschild $\mathrm{BH}$. Indeed, our fits favor $R_{i n}=3 R_{g}$ (see Figure 10), the value one would expect for a maximally (prograde) rotating Kerr BH. This is because, as seen in the figure, a larger $R_{\text {in }}$ will not fit the UV data points. We also performed fits with the color correction of Chiang (2002). This requires a slightly higher disk luminosity $\left(L_{\text {disk }}=2 \times 10^{46} \mathrm{erg} \mathrm{s}^{-1}\right)$, but our results for the inner disk radius remain unchanged. This is of interest since one would expect a BH spin at or near the maximum value if the jet is generated from the Blandford-Znajek mechanism (Blandford \& Znajek 1977). It is also inconsistent with the scenario of Garofalo et al. (2010), where the jets in powerful FR II sources (and presumably FSRQs) are produced by BHs with retrograde spin, requiring that $R_{i n}>6 R_{g}$, while jets in less powerful FR I sources (and presumably BL Lac objects) are produced by BHs with prograde spin. However, there are some caveats regarding the fit of the optical/UV data. The results depend on the $\mathrm{BH}$ mass, although the results for this seem to converge to around $6-8 \times 10^{8} M_{\odot}$.
We also note that if the $\mathrm{BH}$ mass were as low as the one found by Wang et al. (2004), $M_{\mathrm{BH}}=1.5 \times 10^{8} M_{\odot}$, we would not be able to fit the blue bump for this source with a value of the disk luminosity $L_{\text {disk }}$ that is less than the Eddington luminosity. The UV data are often subject to heavy extinction, which could lead to large uncertainty. If the synchrotron component was less steep it could also potentially have a greater contribution to the UV region, masking a larger $R_{i n}$. But in this case the synchrotron emission would over-predict the longer wavelength optical data, so this is unlikely. Finally, the disk model we use is rather simple. It does not include a general relativistic effects such as gravitational Doppler shifts or light bending ( $\mathrm{Li}$ et al. 2005).

Are our modeling results consistent with the observed optical spectra of $4 \mathrm{C}+21.35$ ? Estimates for the luminosity of the broad $\mathrm{H} \beta$ line range from $L_{\mathrm{H} \beta}=2.1 \times 10^{43} \mathrm{erg} \mathrm{s}^{-1}$ (Fan et al. 2006; Tanaka et al. 2011) to the values found from the SDSS spectrum, $L_{\mathrm{H} \beta}=6.3 \times 10^{43} \mathrm{erg} \mathrm{s}^{-1}$ as measured by Shen et al. (2011) and $L_{\mathrm{H} \beta}=5.5 \times 10^{43} \mathrm{erg} \mathrm{s}^{-1}$ by Shaw et al. (2012). Farina et al. (2012) find the line to be quite variable by systematically studying a variety of spectra at different epochs, and their values range from $L_{\mathrm{H} \beta}=3.7 \times 10^{43} \mathrm{erg} \mathrm{s}^{-1}$ to $L_{\mathrm{H} \beta}=6.2 \times 10^{43} \mathrm{erg} \mathrm{s}^{-1}$. Values for the luminosity at $5100 \AA$ are fairly constant if one is careful to exclude the nonthermal component. From the same spectrum, Shen et al. (2011) find $L_{5100}=3.8 \times 10^{45} \mathrm{erg} \mathrm{s}^{-1}$ and Shaw et al. (2012) find $L_{5100}=3.5 \times 10^{45} \mathrm{erg} \mathrm{s}^{-1}$. The estimates by Farina et al. (2012) varies considerably, but their lowest value, with presumably the least amount of contribution from the nonthermal emission, is $L_{5100}=3.5 \times 10^{45} \mathrm{erg} \mathrm{s}^{-1}$. Greene \& Ho (2005) found a tight correlation between $L_{5100}$ and $L_{\mathrm{H} \beta}$, and all the values here, which are around $L_{\mathrm{H} \beta} / L_{5100} \approx 0.01$, are consistent with their correlation. If the total BLR luminosity is $L_{\mathrm{BLR}}=25.3 \times L_{\mathrm{H} \beta}$ (Tanaka et al. 2011), then, using a value $L_{\mathrm{H} \beta}=5 \times 10^{43} \mathrm{erg} \mathrm{s}^{-1}$ that is consistent with the recent measurements (Shen et al. 2011; Shaw et al. 2012; Farina et al. 2012) one gets $L_{\mathrm{BLR}}=1.3 \times 10^{45} \mathrm{erg} \mathrm{s}^{-1}$. Thus, using the disk luminosity for our model, one gets $\xi_{\mathrm{BLR}} \cong L_{\mathrm{BLR}} / L_{\mathrm{disk}} \approx 0.08$, a fairly standard value. Our model fit gives a value of the fraction of the disk radiation reprocessed in the dust torus $\xi_{\text {dust }} \cong L_{\text {dust }} / L_{\text {disk }}=0.34$, again a fairly standard value (e.g., Sikora et al. 2009).

\subsubsection{Jet and Accretion Power}

Our model fits give a total accretion power of $P_{\text {acc }}=$ $L_{\text {disk }} / \eta_{\text {disk }}=1.9 \times 10^{47} \mathrm{erg} \mathrm{s}^{-1}$. If the bolometric isotropic equivalent luminosity from the 2010 June 17 flare is $L_{\text {iso }}=$ $10^{48} \mathrm{erg} \mathrm{s}^{-1}$ (Tanaka et al. 2011) then the radiative efficiency of the flare is

$$
\eta_{j}<\frac{L_{\text {iso }}}{2 \Gamma^{2}\left(P_{j, e}+P_{j, B}\right)} \approx 0.7
$$

(Finke et al. 2008; Sikora et al. 2009; Tanaka et al. 2011, where the factor of 2 takes into account the two-sided jet), which implies a highly radiatively efficient jet. The estimate for the total jet power, $P_{j}=P_{j, e}+P_{j, B}+P_{j, p}$, is a lower limit because it does not include a contribution from protons in the jet $\left(P_{j, p}\right)$, which are likely to be present (e.g., Sikora \& Madejski 2000; Sikora et al. 2009). The jet power contributes a fraction of the total accretion power of

$$
\frac{P_{j, e}+P_{j, B}}{P_{\mathrm{acc}}}=2.3 \times 10^{-3},
$$


although again note that this is a lower limit due to the uncertainty of the proton content. In fact, requiring that $P_{j} / P_{\text {acc }}<1$ gives a constraint on the power in protons in the jet $P_{j, p} \lesssim$ $440 P_{j, e}$. A low $P_{j} / P_{\text {acc }}$ is at odds with the conclusions of Tanaka et al. (2011) who estimate a much higher $P_{j} / P_{\text {acc }}$. The difference is due to their assuming a smaller $\Gamma$ and $\eta_{j}$ than our derived values.

It is interesting to explore the possibility that the flare occurs inside the BLR, and the break in the LAT spectrum is due to $\gamma \gamma$ absorption of $\gamma$-rays with He II Ly photons (continuum and lines) (Poutanen \& Stern 2010; Stern \& Poutanen 2011). Following Tanaka et al. (2011), we find that

$$
L_{\mathrm{He} \text { IILy }} \cong 0.1 L_{\mathrm{H} \mathrm{ILy} \alpha} \cong 4.5 L_{\mathrm{H} \beta} \cong 2.2 \times 10^{44} \mathrm{erg} \mathrm{s}^{-1},
$$

where we have used the value for $L_{\mathrm{H} \beta}$ discussed above. Assuming the typical radius for the He II emission is at a radius $R_{\text {He II }} \cong 0.5 R_{\mathrm{BLR}}$, i.e., at $10^{17} \mathrm{~cm}$, we find that the spectral break one expects from $\gamma \gamma$ absorption with He II Ly photons is

$$
\Delta \Gamma \sim \frac{\tau_{T}(5 \mathrm{GeV})}{4} \cong \frac{\sigma_{T} L_{\mathrm{He} \mathrm{IILy}}}{16 \pi c E_{\mathrm{He} \mathrm{IILy}} R_{\mathrm{He} \mathrm{II}}} \cong 1.2,
$$

significantly larger than the $\Delta \Gamma \cong 0.5$ found in the LAT spectrum for $4 \mathrm{C}+21.35$. The uncertainty in broad emission line luminosities seems to make this approximation a rough estimate. We note that a disk wind model for the BLR (Murray et al. 1995; Chiang \& Murray 1996; Murray \& Chiang 1996) would lower the $\gamma \gamma$ opacity of the BLR, relative to a spherical shell geometry. We tested this possibility, however, and found that the $\gamma \gamma$ opacity remains extremely high, so it is still highly unlikely that MAGIC-detected $\gamma$-ray photons could escape the BLR.

\section{CONCLUSIONS}

$4 \mathrm{C}+21.35$ was detected at VHE by MAGIC on 2010 June 17 during a period of high $\gamma$-ray activity detected by Fermi-LAT. The relatively hard spectrum of the combined HE and VHE spectrum $(\Gamma=2.7 \pm 0.3)$, with no evidence of a cutoff, together with the very rapid variability ( $\sim 10$ minutes) observed by MAGIC challenge standard emission models. We presented multiwavelength observations of the FSRQ 4C +21.35 collected from radio to VHE during 2009-2010. The first hint of a signal at VHE by MAGIC was found on May 3, during a further period of $\gamma$-ray activity observed by Fermi, suggesting a common origin for both the HE and VHE emission during the 2010 April and June episodes.

During 2010 February-June only moderate flux variability was observed in X-rays (a factor of $\sim$ three), with the photon index changing in the range 1.4-2.2 but with no correlation between flux and photon index. A low variability amplitude was observed in UV in the same period, suggesting that the UV is dominated by the accretion disk emission that dilutes the jet emission. It is worth noting that the peak of the UV emission was detected on June 18, but the small increase observed makes it unlikely that the change of the accretion rate is the main driver of the HE and VHE high activity detected by Fermi and MAGIC. The optical light curve shows variable flux density, but no dramatic increase of the activity. Two optical peaks were observed on 2010 April 20 (MJD 55306) and 2010 June 30 (MJD 55377), close in time but not simultaneous with the $\gamma$-ray peaks.

Based on the $15 \mathrm{GHz}$ MOJAVE data, there is no evidence for the ejection of super-luminal knots associated with either of the flares in 2010 April and June. However, Marscher et al. (2012) detected the ejection of a superluminal knot with $43 \mathrm{GHz}$ imaging at a time somewhere between 2010 April 29 and June 3 (MJD 55315-55350), close in time with the first $2010 \gamma$-ray flare (see their Figure 3). We also noted that this knot could be associated with the $\gamma$-ray outburst at around 2010 May 24 (MJD 55340). If the flare occurred at the $43 \mathrm{GHz}$ core, our model implies that the $43 \mathrm{GHz}$ core is about 3 pc from the central BH.

Based on our SED modeling (Section 7.2), we reach the following conclusions.

1. The $\gamma$-ray flares in 2010 April and June cannot have originated from inside the BLR, at least not without invoking some unusual particle transport mechanism (Dermer et al. 2012; Tavecchio et al. 2012).

2. There is some evidence for a rapidly spinning prograde $\mathrm{BH}$ based on the optical emission.

3. The two flaring states and the quiescent state can be modeled by varying only the electron distribution for the source.

The last result, modeling the source by varying only the electron distribution, has also been found for the blazar PKS 0537-441 (D’Ammando et al. 2013). This conclusion is much stronger for PKS 0537-441, since the optical continuum of PKS 0537-441 is not disk-dominated, making its modeling more constraining. Nonetheless, there are clearly sources for which a change in the electron distribution is not sufficient to explain the difference between flaring and quiescent states. For example, to model a strong optical-near infrared flare from PKS 0208-512 with no counterpart in $\gamma$-rays required changing the magnetic field strength (Chatterjee et al. 2013).

Rotation in polarization angles coincident with flares has been observed before in the blazars BL Lac (Marscher et al. 2008), PKS 1510-089 (Marscher et al. 2010; Orienti et al. 2013), and 3C 279 (Abdo et al. 2010). They could be caused by a sudden realignment in the magnetic field due to shock compression, or a curved trajectory taken by the flaring region. A slight increase of the degree of optical polarization but no significant rotation of the polarization angle was observed at the time of the 2010 June HE and VHE flare.

The object $4 \mathrm{C}+21.35$ continues to challenge our understanding of blazar emission mechanisms and the location of the emitting region. Multi-wavelength observations have complemented previous LAT and MAGIC observations to give a more complete picture for this source, although many outstanding questions remain.

The Fermi LAT Collaboration acknowledges generous ongoing support from a number of agencies and institutes that have supported both the development and the operation of the LAT as well as scientific data analysis. These include the National Aeronautics and Space Administration and the Department of Energy in the United States, the Commissariat à l'Energie Atomique and the Centre National de la Recherche Scientifique/Institut National de Physique Nucléaire et de Physique des Particules in France, the Agenzia Spaziale Italiana and the Istituto Nazionale di Fisica Nucleare in Italy, the Ministry of Education, Culture, Sports, Science and Technology (MEXT), High Energy Accelerator Research Organization (KEK) and Japan Aerospace Exploration Agency (JAXA) in Japan, and the K. A. Wallenberg Foundation, the Swedish Research Council and the Swedish National Space Board in Sweden. Additional support for science 
analysis during the operations phase is gratefully acknowledged from the Istituto Nazionale di Astrofisica in Italy and the Centre National d'Études Spatiales in France.

MAGIC Collaboration would like to thank the Instituto de Astrofísica de Canarias for the excellent working conditions at the Observatorio del Roque de los Muchachos in La Palma. The support of the German BMBF and MPG, the Italian INFN, the Swiss National Fund SNF, and the Spanish MICINN is gratefully acknowledged. This work was also supported by the CPAN CSD2007-00042 and MultiDark CSD2009-00064 projects of the Spanish Consolider-Ingenio 2010 programme, by grant 127740 of the Academy of Finland, by the DFG Cluster of Excellence "Origin and Structure of the Universe," by the DFG Collaborative Research Centers SFB823/C4 and SFB876/C3, and by the Polish MNiSzW grant 745/N-HESS-MAGIC/2010/0.

We thank the Swift team for making these observations possible, the duty scientists, and science planners. This research has made use of data from the MOJAVE database that is maintained by the MOJAVE team (Lister et al. 2009, AJ, 137, 3718). The MOJAVE project is supported under NASA-Fermi grant 11-Fermi11-0019. The National Radio Astronomy Observatory is a facility of the National Science Foundation operated under cooperative agreement by Associated Universities, Inc. This work made use of the Swinburne University of Technology software correlator (Deller et al. 2011, PASP, 123, 275), developed as part of the Australian Major National Research Facilities Programme and operated under license. The OVRO 40-m monitoring program is supported in part by NASA grants NNX08AW31G and NNX11A043G, and NSF grants AST-0808050 and AST-1109911. This paper is partly based on observations with the $100 \mathrm{~m}$ telescope of the MPIfR (MaxPlanck-Institut für Radioastronomie) at Effelsberg and the Medicina telescope operated by INAF-Istituto di Radioastronomia. We acknowledge A. Orlati, S. Righini, and the Enhanced Single-dish Control System (ESCS) Development Team. We acknowledge financial contribution from agreement ASI-INAF I/009/10/0. The Submillimeter Array is a joint project between the Smithsonian Astrophysical Observatory and the Academia Sinica Institute of Astronomy and Astrophysics and is funded by the Smithsonian Institution and the Academia Sinica. Data from the Steward Observatory spectropolarimetric monitoring project were used. This program is supported by Fermi Guest Investigator grants NNX08AW56G, NNX09AU10G, and NNX12AO93G. The St. Petersburg University team acknowledges support from Russian RFBR foundation, grants 12-02-00452 and 12-02-31193. The Abastumani team acknowledges financial support of the project FR/638/6-320/12 by the Shota Rustaveli National Science Foundation under contract 31/77. The Metsähovi team acknowledges support from the Academy of Finland to our observing projects (numbers 212656, 210338, 121148, and others). E.R. was partially supported by the Spanish MINECO projects AYA2009-13036-C0202 and AYA2012-38491-C02-01 and by the Generalitat Valenciana project PROMETEO/2009/104, as well as by the COST MP0905 action "Black Holes in a Violent Universe." Y.Y.K. was partly supported by the Russian Foundation for Basic Research (project 13-02-12103) and the Dynasty Foundation. We thank the anonymous referee for useful comments and suggestions. J.F. would like to thank J. Steiner for useful discussions regarding the black hole spin of $4 \mathrm{C}+21.35$. F.D. thanks P. Smith for useful discussions about the polarimetric observations of $4 \mathrm{C}+21.35$.
Facilities: Fermi, MAGIC, Swift, SMA, Metsähovi radio, UMRAO, OVRO:40m, VLBA, Medicina:32m, Crimean, Effelsberg, SO:1.5m

\section{REFERENCES}

Abdo, A. A., Ackermann, M., Ajello, M., et al. 2010, Natur, 463, 919 Abdo, A. A., Ackermann, M., Ajello, M., et al. 2011, ApJ, 730, 101 Abramowicz, A., Acero, F., Aharonian, F., et al. 2013, A\&A, 554, 107 Ackermann, M., Ajello, M., Allefort, A., et al. 2013, ApJS, 209, 34

Ade, P. A. R., Aghanim, N., Armitage-Caplan, C., et al. 2013, A\&A, accepted (arXiv:1303.5088)

Aharonian, F., Akhperjanian, A. G., Bazer-Bachi, A. R., et al. 2007, ApJL, 664, L71

Albert, J., Aliu, E., Anderhub, H., et al. 2007, ApJ, 669, 862

Albert, J., Aliu, E., Anderhub, H., et al. 2008, Sci, 320, 1752

Aleksić, J., Alvarez, E. A., Antonelli, L. A., et al. (the MAGIC Collaboration) 2012, APh, 35, 435

Aleksić, J., Antonelli, L. A., Antoranz, P., et al. (the MAGIC Collaboration) 2011, ApJL, 730, L8

Angelakis, E., Fuhrmann, L., Marchili, N., Krichbaum, T. P., \& Zensus, J. A. 2008, MmSAI, 79, 1042

Atwood, W. B., Abdo, A. A., Ackermann, M., et al. 2009, ApJ, 697, 1071

Baars, J. W. M., Genzel, R., Pauliny-Toth, I. I. K., \& Witzel, A. 1977, A\&A, 61,99

Barthelmy, S. D., Barbier, Louis M., Cummings, Jay R., et al. 2005, SSRv, 120,143

Baumgartner, W. H., Tueller, J., Markwardt, C. B., et al. 2013, ApJS, 207, 19

Bertin, E., \& Arnouts, S. 1996, A\&AS, 117, 393

Blandford, R. D., \& Znajek, R. L. 1977, MNRAS, 179, 433

Burrows, D. N., Hill, J. E., Nousek, J. A., et al. 2005, SSRv, 120, 165

Cash, W. 1979, ApJ, 228, 939

Castignani, G., Haardt, F., Lapi, A., et al. 2013, A\&A, 560, A28

Chatterjee, R., Fossati, G., Urry, C. M., et al. 2013, ApJL, 763, L11

Chiang, J. 2002, ApJ, 572, 79

Chiang, J., \& Murray, N. 1996, ApJ, 466, 705

Cooper, N. J., Lister, M. L., \& Kochanczyk, M. D. 2007, ApJS, 171, 376

Cortina, J. 2012, ATel, 3965

D’Ammando, F., Antolini, E., Tosti, G., et al. 2013, MNRAS, 431, 2481

Dermer, C. D., Finke, J. D., Krug, H., \& Böttcher, M. 2009, ApJ, 692, 32

Dermer, C. D., Murase, K., \& Takami, H. 2012, ApJ, 755, 147

Djorgovski, S. G., Drake, A. J., Mahabal, A. A., et al. 2011, in The First Year of MAXI: Monitoring Variable X-ray Sources, ed. T. Mihara \& N. Kawai (Tokyo: JAXA Special Publ.), 263

Dominguez, A., Primack, J. R., Rosario, D. J., et al. 2011, MNRAS, 410, 2556 Drake, A., Djorgovski, S. G., Mahabal, A., et al. 2009, ApJ, 696, 870

Edelson, R. A., \& Krolik, J. H. 1988, ApJ, 333, 646

Fan, Z., Cao, X., \& Gu, M. 2006, ApJ, 646, 8

Farina, E. P., Decarli, R., Falomo, R., Treves, A., \& Raiteri, C. M. 2012, MNRAS, 424, 393

Finke, J. D., Dermer, C. D., \& Böttcher, M. 2008, ApJ, 686, 181

Finke, J. D., Razzaque, S., \& Dermer, C. D. 2010, ApJ, 712, 238

Fuhrmann, L., Krichbaum, T. P., Witzel, A., et al. 2008, A\&A, 490, 1019

Fuhrmann, L., Zensus, J. A., Krichbaum, T. P., Angelakis, E., \& Readhead, A. C. S. 2007, in AIP Conf. Proc. 921, The First GLAST Symposium, ed. S. Ritz, P. Michelson, \& C. A. Meegan (Melville, NY: AIP), 249

Garofalo, D., Evans, D. A., \& Sambruna, R. M. 2010, MNRAS, 406, 975

Gehrels, N., Chincarini, G., Giommi, P., et al. 2004, ApJ, 611, 1005

Ghisellini, G., Celotti, A., Fossati, G., Maraschi, L., \& Comastri, A. 1998, MNRAS, 301, 451

Ghisellini, G., \& Tavecchio, F. 2008, MNRAS, 386, L28

Giannios, D., Uzdensky, D. A., \& Begelman, M. C. 2009, MNRAS, 395, L29

Greene, J. E., \& Ho, L. C. 2005, ApJ, 630, 122

Gurwell, M. A., Peck, A. B., Hostler, S. R., et al. 2007, in ASP Conf. Ser. 375, From Z-Machines to ALMA: (Sub)Millimeter Spectroscopy of Galaxies, ed. A. J. Baker, J. Glenn, A. I. Harris, J. G. Mangum, \& M. S. Yun (San Francisco, CA: ASP), 234

Hauser, M., Möllenhoff, C., Pühlhofer, G., et al. 2004, AN, 325, 659

Homan, D. C., Ojha, R., Wardle, J. F. C., et al. 2001, ApJ, 549, 840

Jorstad, S. G., Marscher, A. P., Mattox, J. R., et al. 2001, ApJS, 134, 181

Kalberla, P. M. W., Burton, W. B., Hartmann, D., et al. 2005, A\&A, 440, 775

Kaspi, S., Smith, P. S., Netzer, H., et al. 2000, ApJ, 533, 631

Kharb, P., \& Shastri, P. 2004, A\&A, 425, 825

Konigl, A. 1981, ApJ, 243, 700 
Leon-Tavares, J., Chavushyan, V., Patiño-Álvarez, V., et al. 2013, ApJL, 763, L36

Li, L.-X., Zimmerman, E. R., Narayan, R., \& McClintock, J. E. 2005, ApJS, 157,335

Li, T.-P., \& Ma, Y.-Q. 1983, ApJ, 272, 317

Lister, M., Aller, M. F., Aller, H. D., et al. 2009, AJ, 137, 3718

Lister, M., Aller, M. F., Aller, H. D., et al. 2013, AJ, 146, 120

Lombardi, S., Berger, P., Colin, P., et al. 2011, in Proc. 32nd ICRC (Beijing), Vol. 3 HE1.4: Extensive Air Showers and HE Cosmic Rays, 262 (arXiv:1109.6195)

Malmrose, M. P., Marscher, A. P., Jorstad, S. G., Nikutta, R., \& Elitzur, M. 2011, ApJ, 732, 116

Marscher, A. P., \& Jorstad, S. G. 2010, in Fermi meets Jansky - AGN in Radio and Gamma-Rays, ed. T. K. Savolainen, E. Ros, R. W. Porcas, \& J. A. Zensus, MPIfR Bonn, 171 (arXiv:1005.5551)

Marscher, A. P., Jorstad, S. G., Agudo, I., MacDonald, N. R., \& Scott, T. L. 2012, 2012 Fermi \& Jansky Proceedings: Our Evolving Understanding of AGN - eConf C1111101, ed. R. Ojha, D. J. Thompson, \& C. D. Dermer (arXiv:1204.6707)

Marscher, A. P., Jorstad, S. G., D'Arcangelo, F. D., et al. 2008, Natur, 452, 966

Marscher, A. P., Jorstad, S. G., Larionov, V. M., et al. 2010, ApJL, 710, L126

Moralejo, A., Gaug, M., Carmona, E., et al. (the MAGIC Collaboration) 2009, in Proc. of 31st ICRC, ed. J. Szabelski \& M. Giller (Łódź, Poland) (arXiv:0907.0943)

Murray, N., \& Chiang, J. 1996, ApJ, 474, 91

Murray, N., Chiang, J., Grossman, S. A., \& Voit, G. M. 1995, ApJ, 451,498
Nalewajko, K., Begelman, M. C., Cerutti, B., Uzdensky, D. A., \& Sikora, M. 2012, MNRAS, 425, 2519

Nalewajko, K., Giannios, D., Begelman, M. C., Uzdensky, D. A., \& Sikora, M 2011, MNRAS, 413, 333

Nenkova, M., Sirocky, M. M., Nikutta, R., Ivezić, Ž., \& Elitzur, M. 2008, ApJ, 685,160

Orienti, M., Koyama, S., D’Ammando, F., et al. 2013, MNRAS, 428, 2418

Osterbrock, D. E., \& Pogge, R. W. 1987, ApJ, 323, 108

Poutanen, J., \& Stern, B. 2010, ApJL, 717, L118

Richards, J. L., Max-Moerbeck, W., Pavlidou, V., et al. 2011, ApJS, 194, 29

Roming, P. W. A., Kennedy, T. E., Mason, K. O., et al. 2005, SSRv, 120, 95

Saikia, D. J., Wiita, P. J., \& Muxlow, T. W. B. 1993, AJ, 105, 1658

Shakura, N. I., \& Sunyaev, R. A. 1973, A\&A, 24, 337

Shaw, M. S., Romani, R. W., Cotter, G., et al. 2012, ApJ, 748, 49

Shen, Y., Richards, G. T., Strauss, M. A., et al. 2011, ApJS, 194, 45

Sikora, M., \& Madejski, G. 2000, ApJ, 534, 109

Sikora, M., Stawarz, Ł., Moderski, R., Nalewajko, K., \& Madejski, G. M. 2009, ApJ, 704, 38

Smith, P. S., Schmidt, G. D., \& Jannuzi, B. T. 2011, 2011 Fermi Symposium proceedings - eConf C110509, ed. A. Morselli \& the Local Organizing Committee (Rome) (arXiv:1110.6040)

Stern, B. E., \& Poutanen, J. 2011, MNRAS, 417, L11

Tanaka, Y. T., Stawarz, L., Thompson, D. J., et al. 2011, ApJ, 733, 19

Tavecchio, F., Becerra-Gonzales, J., Ghisellini, G., et al. 2011, A\&A, 534, 86

Tavecchio, F., Roncadelli, M., Galanti, G., \& Bonnoli, G. 2012, PhRvD, 86,5036

Teräsranta, H., Tornikoski, M., Mujunen, A., et al. 1998, A\&AS, 132, 305

Wang, J., Luo, B., \& Ho, L. C. 2004, ApJL, 615, L9

White, R. J., \& Peterson, B. A. 1994, PASP, 106, 879 